\title{
Including a phase in the Bethe equations of the Hubbard model
}

\author{
V. Fomin, L. Frappat and E. Ragoucy \\ Laboratoire de Physique Théorique LAPTH, Université de Savoie - CNRS (UMR 5108), \\ BP 110, F-74941 Annecy-le-Vieux Cedex, France \\ E-mail: fomin@lapp.in2p3.fr, frappat@lapp.in2p3.fr, \\ ragoucy@lapp.in2p3.fr
}

Abstract: We compute the Bethe equations of generalized Hubbard models, and study their thermodynamical limit. We argue how they can be connected to the ones found in the context of AdS/CFT correspondence, in particular with the so-called dressing phase problem.

We also show how the models can be interpreted, in condensed matter physics, as integrable multi-leg Hubbard models.

Keywords: Integrable Equations in Physics, Lattice Integrable Models, Bethe Ansatz

ARXIV EPRINT: 1002.1806 


\section{Contents}

1 Introduction $\quad 1$

2 Description of the models 2

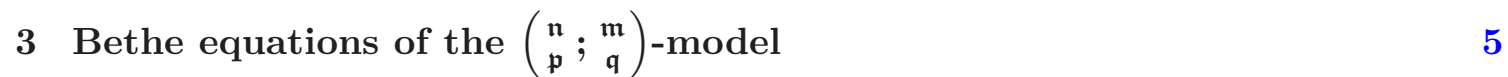

4 Thermodynamical limit $\quad 6$

$\begin{array}{ll}4.1 \text { Simplification of Bethe equations } & 6\end{array}$

4.2 Thermodynamical limit 8

4.3 Ground state 8

$\begin{array}{lr}4.4 \text { String hypothesis } & 9\end{array}$

5 Applications $\quad 11$

5.1 Multi-leg Hubbard models 11

$\begin{array}{ll}5.2 \text { Comparison with AdS/CFT } & 13\end{array}$

$\begin{array}{llr}6 & \text { Conclusion } & 14\end{array}$

$\begin{array}{ll}\text { A Coordinate Bethe ansatz } & \mathbf{1 5}\end{array}$

$\begin{array}{lll}\text { A.1 Coordinate Bethe ansatz, level one } & 15\end{array}$

$\begin{array}{lll}\text { A.2 Auxiliary problem, level two } & 20\end{array}$

\section{Introduction}

The nowadays widely studied Hubbard model was introduced in the sixties [1-3] in relation with strongly correlated electron systems (see $[4,5]$ and references therein for a review on the Hubbard model). In two and three dimensions, the Hubbard models are unfortunately not solved yet and few results have been obtained. In contrast, the one-dimensional Hubbard model was found to be integrable and its Hamiltonian was first diagonalized by means of the coordinate Bethe Ansatz by Lieb and Wu in 1968 [6, 7]. Since then, there have been numerous studies on this model.

Originally described as a model of spin up and down electrons on a one-dimensional lattice with hopping terms and nearest neighbors interactions (resp. kinetic and potential terms of the corresponding Hamiltonian), the Hubbard model has been then generalized in different ways. A particularly interesting method is based on Shastry's construction which was used to reveal the integrable structure of the Hubbard model [8-11]. The main idea is to couple two XX model $R$-matrices through an interaction term depending on the coupling constant of the Hubbard potential. The proof of the Yang-Baxter equation for the obtained Hubbard $R$-matrix was given by Shiroishi and Wadati [12]. 
In this framework, a first generalization to the $g l(\mathfrak{n})$ case was proposed by Maassarani $[13,14]$. The extension to the superalgebraic case $g l(\mathfrak{n} \mid \mathfrak{m})$, mainly motivated by the appearance of the Hubbard model in the context of $N=4$ Yang-Mills theories (see e.g. [15]), was given in [16]. A general approach for deriving (super) Hubbard models was developed in [17]. The construction is based on the decomposition of an arbitrary vector space (possibly infinite dimensional) into a direct sum of two subspaces. The two corresponding orthogonal projectors allow one to define a $R$-matrix of a universal XX model, and then of a Hubbard model using Shastry's trick. The QISM approach ensures the integrability of the models, and the properties of the obtained $R$-matrices lead to local Hubbard-like Hamiltonians.

We continue here the investigations of the one-dimensional integrable generalizations of the Hubbard model started in [17], where Hubbard-like models based on $g l(\mathfrak{n} \mid \mathfrak{m}) \oplus g l\left(\mathfrak{n}^{\prime} \mid \mathfrak{m}^{\prime}\right)$ were introduced and the corresponding Hamiltonians explicitly constructed. The Bethe Ansatz equations were fully derived in the XX case, while only subsectors of the theory were examined in the Hubbard case, due to the complexity of the calculations. A step forward was accomplished in [18], where the case $g l(\mathfrak{n} \mid \mathfrak{m}) \oplus g l(2)$ was investigated and the corresponding BAE determined. In this paper, we give the complete set of Bethe Ansatz equations for general Hubbard-like models. The structure of these BAEs is similar to the one of the usual Hubbard model, however with some (new) phases that depend on some Bethe roots that are quantized. These phases have to be compared to the ones introduced in the context of AdS/CFT correspondence [19, 20]. Hence, we believe that this construction may be seen as a first step in the construction of the integrable model underlying the super-Yang-Mills theories.

Another interesting application of these models relies on their possible interpretation as multi-leg Hubbard models in condensed matter physics. This may be also a new way to tackle the problem of two-dimensional Hubbard models.

The plan of the paper is as follows. In section 2, we remind the construction for general Hubbard models and we set the basic notations used in the paper. Then we present in section 3 the set of Bethe Ansatz equations (BAEs) associated to these models, together with the energies and momenta. In order to make the presentation clearer, the organization and the details of the calculations, based on the coordinate Bethe Ansatz, are postponed in appendix A. The section 4 deals with the thermodynamical limit. The Bethe equations in this limit are derived and the ground state energy is determined. Some applications of these models are presented in section 5. It is first emphasized that the corresponding Hamiltonians can be, after a Jordan-Wigner transformation, interpreted as multi-leg Hubbard models. Another point concerns the possible interpretation of the phases occuring in the considered Bethe equations in relation with the AdS/CFT correspondence. Finally, we conclude in section 6 on open problems.

\section{Description of the models}

Let us here remind the construction for general Hubbard-like models. For a given pair of algebras $(g l(\mathfrak{n}), g l(\mathfrak{m}))$ there are roughly $\left[\frac{(\mathfrak{n}-1)(\mathfrak{m}-1)+1}{2}\right]$ non-trivial inequivalent models, 
corresponding to the choice of a projector in each of the two algebras. These choices are labelled by two integers $(\mathfrak{p}, \mathfrak{q})$, with $1 \leq \mathfrak{p} \leq \mathfrak{n}$ and $1 \leq \mathfrak{q} \leq \mathfrak{m}$, that correspond to the ranks of the projectors.

The Hamiltonian which we deal with in the calculations below is derived from a transfer matrix obtained from the standard procedure used for integrable systems.

At first, we define the $R$-matrix of an XX model based on the algebra $g l(\mathfrak{n})$ :

$$
\begin{aligned}
R_{12}(\lambda) & =\Sigma_{12} P_{12}+\Sigma_{12} \sin \lambda+\left(\mathbb{I} \otimes \mathbb{I}-\Sigma_{12}\right) P_{12} \cos \lambda \in \operatorname{End}\left(\mathbb{C}^{\mathfrak{n}}\right) \otimes \operatorname{End}\left(\mathbb{C}^{\mathfrak{n}}\right) \\
P_{12} & =\sum_{i, j=1}^{\mathfrak{n}} E_{1}^{i j} E_{2}^{j i} \\
\Sigma_{12} & =\sum_{i=1}^{\mathfrak{p}} \sum_{j=\mathfrak{p}+1}^{\mathfrak{n}}\left(E_{1}^{i i} E_{2}^{j j}+E_{1}^{j j} E_{2}^{i i}\right),
\end{aligned}
$$

where $\lambda \in \mathbb{C}$ is the spectral parameter and $\mathfrak{p} \in[1, \mathfrak{n}]$ is a free integer parameter that defines the model. $E_{x}^{i j}$ denote the elementary matrices (with entry 1 at row $i$ and column $j$ and 0 elsewhere) acting in the $x^{t h}$ copy of $\operatorname{End}\left(\mathbb{C}^{\mathfrak{n}}\right)$. The XX-model $R$-matrix $(2.1)$ obeys the Yang-Baxter equation, is unitary and regular.

The definition of the (generalized) Hubbard $R$-matrix uses as basic ingredient the $R$ matrix of the XX model (or its generalization), which are coupled à la Shastry, the coupling constant being related to the potential $\mathfrak{u}$ of the Hubbard model. The two underlying XX models may be based on two different algebras $g l(\mathfrak{n})_{\uparrow}$ and $g l(\mathfrak{m})_{\downarrow}$ and depend on two different integers $\mathfrak{p}$ and $\mathfrak{q}$ [17]. Let us introduce the corresponding sets of integers

$\mathcal{N}_{\uparrow}=\{1,2, \ldots, \mathfrak{p}\}, \overline{\mathcal{N}}_{\uparrow}=\{\mathfrak{p}+1, \ldots, \mathfrak{n}\} \quad$ and $\quad \mathcal{N}_{\downarrow}=\{1,2, \ldots, \mathfrak{q}\}, \overline{\mathcal{N}}_{\downarrow}=\{\mathfrak{q}+1, \ldots, \mathfrak{m}\}$.

The $R$-matrix of the Hubbard model based on the pair $(g l(\mathfrak{n}), \mathfrak{p} ; g l(\mathfrak{m}), \mathfrak{q})$ is given by

$$
R_{12}^{\uparrow \downarrow}\left(\lambda_{1}, \lambda_{2}\right)=R_{12}^{\uparrow}\left(\lambda_{12}\right) R_{12}^{\downarrow}\left(\lambda_{12}\right)+\frac{\sin \left(\lambda_{12}\right)}{\sin \left(\lambda_{12}^{\prime}\right)} \tanh \left(h_{12}^{\prime}\right) R_{12}^{\uparrow}\left(\lambda_{12}^{\prime}\right) C_{\uparrow 1} R_{12}^{\downarrow}\left(\lambda_{12}^{\prime}\right) C_{\downarrow 1}
$$

where $\lambda_{12}=\lambda_{1}-\lambda_{2}, \lambda_{12}^{\prime}=\lambda_{1}+\lambda_{2}$ and we introduced the diagonal matrix $C_{\alpha}(\alpha=\uparrow$ or $\downarrow)$ :

$$
C_{\alpha}=\sum_{j \in \mathcal{N}_{\alpha}} E_{\alpha}^{j j}-\sum_{\bar{j} \in \overline{\mathcal{N}}_{\alpha}} E_{\alpha}^{\overline{j \jmath}}
$$

The coupling $h_{12}^{\prime}=h\left(\lambda_{1}\right)+h\left(\lambda_{2}\right)$ is based on the function $h(\lambda)$ such that

$$
\sinh (2 h)=\frac{\mathfrak{u}}{4} \sin (2 \lambda) .
$$

The $R$-matrix (2.5) is symmetric, regular and satisfies the unitary relation. Moreover, when the relation (2.7) holds, the $R$-matrix (2.5) satisfies the Yang-Baxter equation:

$$
R_{12}^{\uparrow \downarrow}\left(\lambda_{1}, \lambda_{2}\right) R_{13}^{\uparrow \downarrow}\left(\lambda_{1}, \lambda_{3}\right) R_{23}^{\uparrow \downarrow}\left(\lambda_{2}, \lambda_{3}\right)=R_{23}^{\uparrow \downarrow}\left(\lambda_{2}, \lambda_{3}\right) R_{13}^{\uparrow \downarrow}\left(\lambda_{1}, \lambda_{3}\right) R_{12}^{\uparrow \downarrow}\left(\lambda_{1}, \lambda_{2}\right) .
$$

Being equipped with an $R$-matrix with all required properties, we can proceed to define the corresponding quantum integrable system, by performing the following steps: monodromy matrix, transfer matrix and Hamiltonian. The $L$-site monodromy matrix is given

$$
T_{a<b_{1} \ldots b_{L}>}(\lambda)=R_{a b_{1}}^{\uparrow \downarrow}(\lambda, 0) \ldots R_{a b_{L}}^{\uparrow \downarrow}(\lambda, 0)
$$


and its transfer matrix is the (super)trace in the auxiliary space:

$$
t(\lambda)=\operatorname{tr}_{a} T_{a<b_{1} \ldots b_{L}>}(\lambda) .
$$

Then the generalized Hubbard Hamiltonian reads

$$
H=\left.\frac{d}{d \lambda} \ln t(\lambda)\right|_{\lambda=0}=\sum_{x=1}^{L} H_{x, x+1}
$$

with

$$
H_{x, x+1}=(\Sigma P)_{\uparrow x, x+1}+(\Sigma P)_{\downarrow x, x+1}+\frac{\mathfrak{u}}{4} C_{\uparrow x} C_{\downarrow x},
$$

where we have used periodic boundary conditions.

We consider generalized $(g l(\mathfrak{n}), \mathfrak{p} ; g l(\mathfrak{m}), \mathfrak{q})$ models, containing thus four different types of particles $\pi \uparrow, \bar{\pi} \uparrow, \pi \downarrow$ and $\bar{\pi} \downarrow$, each type being 'colored': the $\pi \uparrow$-particles have 'colors' $1 \uparrow, 2 \uparrow, \ldots, \mathfrak{p} \uparrow$; the $\bar{\pi} \uparrow$-particles have 'colors' $(\mathfrak{p}+1) \uparrow, \ldots, \mathfrak{n} \uparrow$, while the 'colors' for $\pi \downarrow$ and $\bar{\pi} \downarrow$-particles are $1 \downarrow, \ldots, \mathfrak{q} \downarrow$ and $(\mathfrak{q}+1) \downarrow, \ldots, \mathfrak{m} \downarrow$ respectively.

The $L$-site Hamiltonian is given by

$$
H=\sum_{x=1}^{L}\left((\Sigma P)_{\uparrow x, x+1}+(\Sigma P)_{\downarrow x, x+1}+\frac{\mathfrak{u}}{4} C_{\uparrow x} C_{\downarrow x}\right), \quad \bmod L
$$

where $\mathfrak{u}>0$ and

$$
\begin{aligned}
(\Sigma P)_{\uparrow x, x+1} & =\sum_{i \in \mathcal{N}_{\uparrow}} \sum_{j \in \overline{\mathcal{N}}_{\uparrow}}\left(E_{\uparrow x}^{i j} E_{\uparrow x+1}^{j i}+E_{\uparrow x}^{j i} E_{\uparrow x+1}^{i j}\right), \\
(\Sigma P)_{\downarrow x, x+1} & =\sum_{i \in \mathcal{N}_{\downarrow}} \sum_{j \in \overline{\mathcal{N}}_{\downarrow}}\left(E_{\downarrow x}^{i j} E_{\downarrow x+1}^{j i}+E_{\downarrow x}^{j i} E_{\downarrow x+1}^{i j}\right), \\
C_{\uparrow x} & =\sum_{i \in \mathcal{N}_{\uparrow}} E_{\uparrow x}^{i i}-\sum_{j \in \overline{\mathcal{N}}_{\uparrow}} E_{\uparrow x}^{j j} ; \quad C_{\downarrow x}=\sum_{i \in \mathcal{N}_{\downarrow}} E_{\downarrow x}^{i i}-\sum_{j \in \overline{\mathcal{N}}_{\downarrow}} E_{\downarrow x}^{j j} .
\end{aligned}
$$

The corresponding system will be called a $(g l(\mathfrak{n}), \mathfrak{p} ; g l(\mathfrak{m}), \mathfrak{q})$ model, or a $\left(\begin{array}{c}\mathfrak{n} \\ \mathfrak{p}\end{array} ; \mathfrak{\mathfrak { q }}\right)$-model. Note that the $\left(\begin{array}{c}\mathfrak{n} \\ \mathfrak{n}-\mathfrak{p}\end{array} ; \mathfrak{m}-\mathfrak{q}\right.$ bard model is the $\left(\begin{array}{l}2 \\ 1\end{array} ; \begin{array}{l}2 \\ 1\end{array}\right)$-model. The models introduced by Maassarani $[13,14]$ are the $\left(\begin{array}{l}\mathfrak{n} \\ 1\end{array} ; \begin{array}{c}\mathfrak{m} \\ 1\end{array}\right)$-ones. Generalizations to the superalgebra case as done in [17] will be noted

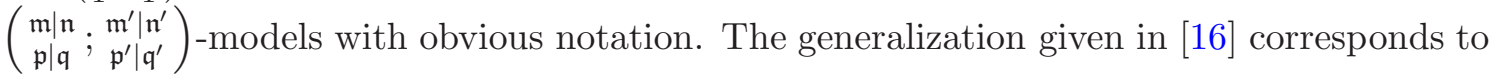
$\left(\begin{array}{c}\mathfrak{m} \mid \mathfrak{n} \\ 1 \mid 1\end{array} ; \begin{array}{c}\mathfrak{m}^{\prime} \mid \mathfrak{n}^{\prime} \\ 1 \mid 1\end{array}\right)$-models. The symmetry algebra of the $\left(\begin{array}{c}\mathfrak{n} \\ \mathfrak{p}\end{array} ; \mathfrak{q}\right)$-model is a $g l(\mathfrak{p}) \oplus g l(\mathfrak{n}-\mathfrak{p}) \oplus$ $g l(\mathfrak{q}) \oplus g l(\mathfrak{m}-\mathfrak{q})$ algebra.

Remark that one can add to the Hamiltonian chemical potentials

$$
\sum_{x=1}^{L}\left(\mu_{\downarrow} \sum_{i \in \mathcal{N}_{\downarrow}} E_{\downarrow x}^{i i}+\bar{\mu}_{\downarrow} \sum_{j \in \overline{\mathcal{N}}_{\downarrow}} E_{\downarrow x}^{j j}+\mu_{\uparrow} \sum_{i \in \mathcal{N}_{\uparrow}} E_{\uparrow x}^{i i}+\bar{\mu}_{\uparrow} \sum_{j \in \overline{\mathcal{N}}_{\uparrow}} E_{\uparrow x}^{j j}\right)
$$

without perturbing integrability nor symmetry. 


\section{Bethe equations of the $\left(\begin{array}{c}\mathfrak{n} \\ \mathfrak{p}\end{array} ; \begin{array}{c}\mathfrak{m} \\ \mathfrak{q}\end{array}\right)$-model}

We present in this section the main result of the paper, namely the Bethe Ansatz Equations of the model under consideration, see Hamiltonian (2.13). The details of the computation are postponed in the appendix and can be skipped in a first reading of the paper.

The spectrum of the generalized $\left(\begin{array}{c}\mathfrak{n} \\ \mathfrak{p}\end{array} ; \mathfrak{q}\right)$-Hubbard model is

$$
E=\frac{\mathfrak{u}}{4}\left(L-2 N_{\bar{\pi}}\right)+2 \sum_{l \in \mathcal{M}_{\bar{\pi} \uparrow} \cup \mathcal{M}_{\bar{\pi} \downarrow}} \cos \left(k_{l}\right)
$$

where the Bethe roots $k_{i}$ are parameters solution of the Bethe Ansatz equations (see below) and $L$ is the number of sites. To present them, we introduce integers $0 \leq K \leq N \leq L$, $N_{\uparrow \pi}, N_{\uparrow \bar{\pi}}, N_{\downarrow \pi}, N_{\downarrow \bar{\pi}} \geq 0$ such that $K=N_{\uparrow \pi}+N_{\uparrow \bar{\pi}}+N_{\downarrow \pi}+N_{\downarrow \bar{\pi}}$, and sets of integers

$$
\begin{aligned}
\mathcal{M}_{\pi_{\uparrow}} & =\left\{1, \ldots, N_{\uparrow \pi}\right\}, \quad \mathcal{M}_{\bar{\pi}_{\uparrow}}=\left\{N_{\uparrow \pi}+1, \ldots, N_{\uparrow \pi}+N_{\uparrow \bar{\pi}}\right\} \\
\mathcal{M}_{\pi_{\downarrow}} & =\left\{N_{\uparrow \pi}+N_{\uparrow \bar{\pi}}+1, \ldots, N_{\uparrow \pi}+N_{\uparrow \bar{\pi}}+N_{\downarrow \pi}\right\}, \\
\mathcal{M}_{\bar{\pi}_{\downarrow}} & =\left\{N_{\uparrow \pi}+N_{\uparrow \bar{\pi}}+N_{\downarrow \pi}+1, \ldots, K\right\} ; \\
\mathbb{A} & =\mathbb{A}_{\pi \uparrow} \cup \mathbb{A}_{\pi \downarrow}=\left\{a_{1}, a_{2}, \ldots, a_{N_{\uparrow \pi}}\right\} \cup\left\{a_{N_{\uparrow \pi}+N_{\uparrow \pi}+1}, a_{N_{\uparrow \pi}+N_{\uparrow \bar{\pi}}+2}, \ldots, a_{N_{\uparrow \pi}+N_{\uparrow \pi}+N_{\downarrow}}\right\}
\end{aligned}
$$

The integers $a_{j}$ are such that $a_{i} \neq a_{j}$ for $i \in \mathcal{M}_{\pi \uparrow}, j \in \mathcal{M}_{\pi \downarrow}$ and

$$
1 \leq a_{1}<a_{2}<\ldots<a_{N_{\uparrow \pi}} \leq N \quad, \quad 1 \leq a_{N_{\uparrow \pi}+N_{\uparrow \bar{\pi}}+1}<\ldots<a_{N_{\uparrow \pi}+N_{\uparrow \bar{\pi}}+N_{\downarrow}} \leq N
$$

Then, Bethe Ansatz equations are

$$
\begin{aligned}
e^{i k_{j}\left(L-N_{\uparrow \bar{\pi}}\right)} & =(-1)^{N_{\uparrow \pi}-1} e^{2 \pi i \frac{m_{\uparrow \pi}}{N_{\uparrow \pi}}}, m_{\uparrow \pi}=1, \ldots, N_{\uparrow \pi} \quad \text { for } \quad j \in \mathbb{A}_{\pi \uparrow}, \\
e^{i k_{j}\left(L-N_{\downarrow}-N_{\downarrow \mathfrak{m}}\right)} & =(-1)^{N_{\downarrow \pi}-1} e^{2 \pi i \frac{m_{\downarrow \pi}}{N_{\downarrow}}}, m_{\downarrow \pi}=1, \ldots, N_{\downarrow \pi} \quad \text { for } \quad j \in \mathbb{A}_{\pi \downarrow}, \\
e^{i k_{j} L} & =(-1)^{N_{\downarrow \bar{\pi}}+N_{\downarrow \mathfrak{m}}-1} \prod_{m \in \mathcal{M}_{\pi \downarrow}} e^{-i k_{a m}} \prod_{l \in \mathcal{M}_{\bar{\pi} \uparrow}} \frac{i \sin k_{j}+i \lambda_{l}+\frac{\mathfrak{u}}{4}}{i \sin k_{j}+i \lambda_{l}-\frac{\mathfrak{u}}{4}} \prod_{m^{\prime} \in \mathcal{M}_{\bar{\pi} \downarrow}} b_{m^{\prime}},
\end{aligned}
$$

for $j \in[1, N] \backslash \mathbb{A}$

$$
b_{l}^{N_{\downarrow \bar{\pi}}+N_{\downarrow \mathfrak{m}}}=e^{\frac{2 \pi i}{N_{\downarrow}} \sum_{j=1}^{N_{\downarrow \bar{\pi}}-N_{\downarrow}(\mathfrak{m}-1)} n_{j}}, \quad l \in \mathcal{M}_{\bar{\pi} \downarrow}, \quad \arg \left(b_{l}\right)<\arg \left(b_{l+1}\right)
$$

with $1 \leq n_{1}<\ldots<n_{N_{\downarrow}-N_{\downarrow}(\mathfrak{m}-1)} \leq N_{\downarrow \bar{\pi}}$,

$$
\begin{aligned}
& \Lambda \prod_{\substack{l \in \mathcal{M}_{\bar{\pi} \uparrow} \\
l \neq m}} \frac{i \lambda_{m}-i \lambda_{l}+\frac{\mathfrak{u}}{2}}{i \lambda_{m}-i \lambda_{l}-\frac{\mathfrak{u}}{2}}=\prod_{\substack{l=1 \\
l \notin \mathbb{A}}}^{N} \frac{i \sin k_{l}+i \lambda_{m}+\frac{\mathfrak{u}}{4}}{i \sin k_{l}+i \lambda_{m}-\frac{\mathfrak{u}}{4}}, \quad \text { for } \quad m \in \mathcal{M}_{\uparrow \bar{\pi}} \\
& \Lambda=(-1)^{N-N_{\pi \uparrow}-N_{\pi \downarrow}} \prod_{m \in \mathcal{M}_{\pi \downarrow}} e^{i k_{a_{m}}} \prod_{l \in \mathcal{M}_{\bar{\pi} \downarrow}} b_{l}^{-1} \prod_{m^{\prime} \in \mathcal{M}_{\pi \uparrow}} e^{-i k_{a_{m^{\prime}}}} e^{\frac{2 \pi i}{N_{\uparrow \bar{\pi}}} \sum_{l^{\prime}=1}^{N_{\uparrow \bar{\pi}}-N_{\uparrow \mathfrak{n}}} \bar{n}_{l^{\prime}}},
\end{aligned}
$$

with $1 \leq \bar{n}_{1}<\ldots<\bar{n}_{N_{\uparrow \bar{\pi}}-N_{\uparrow \mathfrak{n}}} \leq N_{\uparrow \bar{\pi}}$. 
We have chosen $e^{1 \uparrow} \otimes e^{1 \downarrow}$ as the reference state at the first level of the Bethe ansatz (see appendix A), so that all states with $e^{2 \uparrow}, \ldots, e^{\mathfrak{n} \uparrow}$ and $e^{2 \downarrow}, \ldots, e^{\mathfrak{m} \downarrow}$ appear as excitations ("particles") above the reference state. $N$ is total number of such particles.

The parameter $N_{\uparrow i}$ is the number of $i \uparrow$ particles for $i=2, \ldots, \mathfrak{n}$ and $N_{\downarrow j}$ is the number of $j \downarrow$ particles for $j=3, \ldots, \mathfrak{m} . N_{\uparrow \pi}=\sum_{i=2}^{\mathfrak{p}} N_{\uparrow i}$ and $N_{\downarrow \pi}=\sum_{i=2}^{\mathfrak{q}} N_{\downarrow i}$ count the number of $\pi$-particles with spin up and spin down respectively. $N_{\uparrow \bar{\pi}}=\sum_{i=\mathfrak{p}+1}^{\mathfrak{n}} N_{\uparrow i}$ counts the number of spin up $\bar{\pi}$-particles, while $N_{\downarrow \bar{\pi}}=\sum_{i=\mathfrak{q}+1}^{\mathfrak{m}-1} N_{\downarrow i}$ counts the number of spin down $\bar{\pi}$-particles that are not of type $\mathfrak{m} \downarrow$. The reason for this latter choice will become clear in the following. In the same way, $K=N_{\uparrow \pi}+N_{\uparrow \bar{\pi}}+N_{\downarrow \pi}+N_{\downarrow \bar{\pi}}$ is the total number of particles that are not of type $\mathfrak{m} \downarrow$.

For given integers $N_{\uparrow \pi}, N_{\uparrow \bar{\pi}}, N_{\downarrow \pi}$ and $N_{\downarrow \bar{\pi}}$, the phases $b_{j}$ and the integers $n_{j}$ and $\bar{n}_{j}$ correspond to the different "colors" that can have particles of a given type $(\uparrow \pi, \uparrow \bar{\pi}, \downarrow \pi$ or $\downarrow \bar{\pi}$ types).

The integers $a_{j}$ (entering in the sets $\mathbb{A}_{\pi \uparrow}$ and $\mathbb{A}_{\pi \downarrow}$ ) define the order between momenta of the $\uparrow \pi$ and $\downarrow \pi$ particles. This order is preserved (up to a cyclic permutation) by the action of the Hamiltonian on the wavefunction.

\section{Thermodynamical limit}

The Bethe equations given in section 3 differ from the (usual) Hubbard model's ones by some phases. In this section, we study them in more detail and look for their thermodynamical limit $L \rightarrow \infty$.

\subsection{Simplification of Bethe equations}

The Bethe equations (3.7)-(3.11) can be partly solved for Bethe roots $k_{j}$ with $j \in \mathbb{A}_{\pi \uparrow} \cup \mathbb{A}_{\pi \downarrow}$ and $b_{l}$ with $l \in \mathcal{M}_{\bar{\pi} \downarrow}$ :

$$
\begin{aligned}
& k_{j}= \frac{2 \pi}{L-N_{\uparrow \bar{\pi}}}\left(\frac{N_{\uparrow \pi}-1}{2}+\frac{m_{\uparrow \pi}}{N_{\uparrow \pi}}+I_{j}^{\uparrow \pi}\right), \quad \text { for } \quad j \in \mathbb{A}_{\pi \uparrow}, \\
& m_{\uparrow \pi}=1, \ldots, N_{\uparrow \pi} \text { and } 1 \leq I_{1}^{\uparrow \pi}<\ldots<I_{N_{\uparrow \pi}^{\uparrow \pi}} \leq L-N_{\uparrow \bar{\pi}} \\
& k_{j}= \frac{2 \pi}{L-N_{\downarrow \bar{\pi}}-N_{\downarrow \mathfrak{m}}}\left(\frac{N_{\downarrow \pi}-1}{2}+\frac{m_{\downarrow \pi}}{N_{\downarrow \pi}}+I_{j}^{\downarrow \pi}\right), \quad \text { for } \quad j \in \mathbb{A}_{\pi \downarrow}, \\
& m_{\downarrow \pi}=1, \ldots, N_{\downarrow \pi} \text { and } 1 \leq I_{1}^{\downarrow \pi}<\ldots<I_{N_{\downarrow \pi}}^{\downarrow \pi} \leq L-N_{\downarrow \bar{\pi}}-N_{\downarrow \mathfrak{m}} \\
& \ln b_{l}= \frac{2 \pi i}{N_{\downarrow \bar{\pi}}+N_{\downarrow \mathfrak{m}}}\left(\sum_{j=1}^{N_{\downarrow \bar{\pi}}-N_{\downarrow \mathfrak{m}-1}} \frac{n_{j}}{N_{\downarrow \bar{\pi}}}+I_{l}^{\downarrow \bar{\pi}}\right), \quad l \in \mathcal{M}_{\bar{\pi} \downarrow}, \\
& 1 \leq n_{1}<\ldots<n_{N_{\downarrow \bar{\pi}}-N_{\downarrow(\mathfrak{m}-1)}} \leq N_{\downarrow \bar{\pi}} ; 1 \leq I_{1}^{\downarrow \bar{\pi}}<\ldots<I_{N_{\downarrow \bar{\pi}}}^{\downarrow \bar{\pi}} \leq N_{\downarrow \bar{\pi}}+N_{\downarrow \mathfrak{m}} \\
& e^{i k_{j} L}= e^{2 \pi i \Phi} \prod_{l \in \mathcal{M}_{\bar{\pi} \uparrow}} \frac{i \sin k_{j}+i \lambda_{l}+\frac{\mathfrak{u}}{4}}{i \sin k_{j}+i \lambda_{l}-\frac{\mathfrak{u}}{4}}, \quad \text { for } \quad j \in[1, N] \backslash \mathbb{A} \\
& \prod_{l=1}^{N} \frac{i \sin k_{l}+i \lambda_{m}+\frac{\mathfrak{u}}{4}}{i \sin k_{l}+i \lambda_{m}-\frac{\mathfrak{u}}{4}}=e^{2 \pi i \Psi} \prod_{\substack{l \in \mathcal{M}_{\bar{\pi} \uparrow} \\
l \neq m}} \frac{i \lambda_{m}-i \lambda_{l}+\frac{\mathfrak{u}}{2}}{i \lambda_{m}-i \lambda_{l}-\frac{\mathfrak{u}}{2}}, \quad \text { for } \quad m \in \mathcal{M}_{\bar{\pi} \uparrow}
\end{aligned}
$$


where the phases $\Phi$ and $\Psi$ are defined by

$$
\begin{aligned}
\Phi \equiv & \frac{N_{\downarrow \bar{\pi}}+N_{\downarrow \mathfrak{m}}-1}{2}-\left(\frac{N_{\downarrow \pi}-1}{2}+\frac{m_{\downarrow \pi}}{N_{\downarrow \pi}}\right) \frac{N_{\downarrow \pi}}{L-N_{\downarrow \bar{\pi}}-N_{\downarrow \mathfrak{m}}} \\
& -\sum_{j=1}^{N_{\downarrow \pi}} \frac{I_{j}^{\downarrow \pi}}{L-N_{\downarrow \bar{\pi}}-N_{\downarrow \mathfrak{m}}}+\sum_{j=1}^{N_{\downarrow \bar{\pi}}-N_{\downarrow}(\mathfrak{m}-1)} \frac{n_{j}}{N_{\downarrow \bar{\pi}}+N_{\downarrow \mathfrak{m}}}+\sum_{j=1}^{N_{\downarrow \bar{\pi}}} \frac{I_{j}^{\downarrow \bar{\pi}}}{N_{\downarrow \bar{\pi}}+N_{\downarrow \mathfrak{m}}}
\end{aligned}
$$

and

$$
\begin{aligned}
\Psi \equiv \sum_{\sigma=\uparrow, \downarrow} & \left((-1)^{\delta_{\sigma, \downarrow}}\left(\frac{N_{\sigma \pi}-1}{2}+\frac{m_{\sigma \pi}}{N_{\sigma \pi}}\right) \frac{N_{\sigma \pi}}{L-N_{\sigma \bar{\pi}}-\delta_{\sigma, \downarrow} N_{\downarrow \mathfrak{m}}}+\sum_{j=1}^{N_{\sigma \pi}} \frac{I_{j}^{\sigma \pi}}{L-N_{\sigma \bar{\pi}}-\delta_{\sigma, \downarrow} N_{\downarrow \mathfrak{m}}}\right) \\
& +\frac{N_{\uparrow \bar{\pi}}+N_{\downarrow \bar{\pi}}+N_{\downarrow \mathfrak{m}}}{2}-\sum_{j=1}^{N_{\downarrow \bar{\pi}}-N_{\downarrow \mathfrak{m}-1}} \frac{n_{j}}{N_{\downarrow \bar{\pi}}+N_{\downarrow \mathfrak{m}}}+\sum_{j=1}^{N_{\downarrow \bar{\pi}}} \frac{I_{j}^{\downarrow \bar{\pi}}}{N_{\downarrow \bar{\pi}}+N_{\downarrow \mathfrak{m}}}+\sum_{j=1}^{N_{\uparrow \bar{\pi}}-N_{\uparrow \mathfrak{n}}} \frac{\bar{n}_{j}}{N_{\uparrow \bar{\pi}}}
\end{aligned}
$$

with $1 \leq \bar{n}_{1}<\ldots<\bar{n}_{N_{\uparrow \bar{\pi}}-N_{\uparrow \mathfrak{n}}} \leq N_{\uparrow \bar{\pi}}$.

We recall that

$$
\mathbb{A}=\mathbb{A}_{\pi \uparrow} \cup \mathbb{A}_{\pi \downarrow}=\left\{a_{1}, a_{2}, \ldots, a_{N_{\uparrow \pi}}\right\} \cup\left\{a_{N_{\uparrow \pi}+N_{\uparrow \bar{\pi}}+1}, a_{N_{\uparrow \pi}+N_{\uparrow \bar{\pi}}+2}, \ldots, a_{N_{\uparrow \pi}+N_{\uparrow \bar{\pi}}+N_{\downarrow}}\right\}
$$

where the integers $a_{i}$ are ordered according to the inequalities

$$
1 \leq a_{1}<a_{2}<\ldots<a_{N_{\uparrow \pi}} \leq N \text { and } 1 \leq a_{N_{\uparrow \pi}+N_{\uparrow \bar{\pi}}+1}<a_{N_{\uparrow \pi}+N_{\uparrow \bar{\pi}}+2}<\ldots<a_{N_{\uparrow \pi}+N_{\uparrow \bar{\pi}}+N_{\downarrow \pi}} \leq N
$$

and $a_{i} \neq a_{j}$ for $i \in \mathcal{M}_{\pi \uparrow}, j \in \mathcal{M}_{\pi \downarrow}$.

In order to coincide with the results in $[21,22]$, we now slightly change the notations

$$
\lambda_{i} \rightarrow-\Lambda_{i} \quad \text { and } \quad \mathfrak{u} \rightarrow-4 U
$$

as well as the sign of the Hamiltonian for the energy to be equal to

$$
E=-\mathrm{U}\left(L-2 N_{\bar{\pi}}\right)-2 \sum_{l \in \mathcal{M}_{\bar{\pi} \uparrow} \cup \mathcal{M}_{\bar{\pi} \downarrow}} \cos \left(k_{l}\right)
$$

The ground state of the model is given by the real values of the Bethe roots $k_{j}$ and $\Lambda_{i}$.

Following Lieb and $\mathrm{Wu}[6,7]$, we take the logarithm of the Bethe equations (4.4) and (4.5):

$$
\begin{aligned}
& k_{j} L=2 \pi\left(\Phi+I_{j}\right)+\sum_{i=1}^{N_{\uparrow \bar{\pi}}} \theta\left(\frac{\Lambda_{i}-\sin k_{j}}{U}\right), \quad j \in[1, N] \backslash \mathbb{A} \\
& \sum_{\substack{j=1 \\
j \notin \mathbb{A}}}^{N} \theta\left(\frac{\Lambda_{i}-\sin k_{j}}{U}\right)=2 \pi\left(J_{i}+\Psi\right)+\sum_{k=1}^{N_{\uparrow \bar{\pi}}} \theta\left(\frac{\Lambda_{i}-\Lambda_{k}}{2 U}\right), i \in \mathcal{M}_{\bar{\pi} \uparrow}
\end{aligned}
$$

where $\theta(x)=2 \arctan (x) \in]-\pi, \pi]$ and we take the cut off for the logarithm as $\frac{1}{i} \log \left(\frac{x+i}{x-i}\right)=\pi-\theta(x) \in[0,2 \pi[$.

The quantum number $I_{j}$ is integer or half-integer depending whether $N_{\uparrow \bar{\pi}}+N_{\downarrow \bar{\pi}}+$ $N_{\downarrow \mathfrak{m}}-1$ is even or odd, similarly $J_{i}$ is integer (half-integer) for $N_{\uparrow \bar{\pi}}+1$ even (odd). 


\subsection{Thermodynamical limit}

We consider the thermodynamic limit $L \rightarrow \infty$ where the particle densities $\frac{N_{\sigma \bar{\pi}}}{L}, \frac{N_{\sigma \pi}}{L}$ are kept fixed $(\sigma=\uparrow, \downarrow)$. Considering the phases $\Phi$ and $\Psi$ in the thermodynamical limit the ratios $\frac{\Phi}{L}$ and $\frac{\Psi}{L}$ do not vanish and depend on the particle densities.

In this limit, the real numbers $k_{j}$ and $\Lambda_{i}$ are close one to each other: $k_{j+1}-k_{j} \rightarrow 0$, $\Lambda_{i+1}-\Lambda_{i} \rightarrow 0$ with $L \rightarrow \infty$. They are distributed between $-Q(\Phi)$ and $Q(\Phi) \leq \pi$ and $-B(\Psi)$ and $B(\Psi)<\infty$ for some $Q(\Phi)$ and $B(\Psi)$. In the small intervals $d k$ and $d \Lambda$, the numbers of $k_{j}$ and $\Lambda_{i}$ are $L \rho(k) d k$ and $L \sigma(\Lambda) d \Lambda$ respectively, where $\rho(k)$ and $\sigma(\Lambda)$ are density functions to be determined. They are normalized as follows:

$$
\int_{-Q(\Phi)}^{Q(\Phi)} \rho(k) d k=\frac{N-N_{\uparrow \pi}-N_{\downarrow \pi}}{L} \text { and } \int_{-B(\Psi)}^{B(\Psi)} \sigma(\Lambda) d \Lambda=\frac{N_{\uparrow \bar{\pi}}}{L}
$$

The counting functions $I(k)$ and $J(\Lambda)$ are defined as usual from $I_{j}$ and $J_{i}$ in the continuum limit:

$$
\begin{aligned}
& I(k)=\frac{k L}{2 \pi}-\Phi-\frac{1}{2 \pi} \int_{-B(\Psi)}^{B(\Psi)} d \Lambda \sigma(\Lambda) \theta\left(\frac{\Lambda-\sin k}{U}\right) \\
& J(\Lambda)=\frac{1}{2 \pi} \int_{-Q(\Phi)}^{Q(\Phi)} d k \rho(k) \theta\left(\frac{\Lambda-\sin k}{U}\right)-\Psi-\frac{1}{2 \pi} \int_{-B(\Psi)}^{B(\Psi)} d \Lambda^{\prime} \sigma\left(\Lambda^{\prime}\right) \theta\left(\frac{\Lambda-\Lambda^{\prime}}{2 U}\right)
\end{aligned}
$$

These functions are also such that $I\left(k_{j}\right)=I_{j}$ and $J\left(\Lambda_{i}\right)=J_{i}$.

Since $I(k+d k)-I(k)$ counts the number of $k$ values between $k$ and $k+d k$, we have $\frac{d I(k)}{d k}=L \rho(k)$, and similarly, $\frac{d J(\Lambda)}{d \Lambda}=L \sigma(\Lambda)$.

Now taking the derivatives of (4.13), and considering the fact that the phases $\Phi$ and $\Psi$ do not depend on the Bethe roots $k$ or $\Lambda$, we get the equations on densities, which are the same as Lieb and $\mathrm{Wu}$ in $[6,7]$ :

$$
\begin{aligned}
1 & =2 \pi \rho(k)-\frac{\cos k}{U} \int_{-B}^{B} d \Lambda \sigma(\Lambda) \theta^{\prime}\left(\frac{\Lambda-\sin k}{U}\right) \\
\int_{-Q}^{Q} d k \frac{\rho(k)}{U} \theta^{\prime}\left(\frac{\Lambda-\sin k}{U}\right) & =2 \pi \sigma(\Lambda)+\int_{-B}^{B} d \Lambda^{\prime} \frac{\sigma(\Lambda)}{2 U} \theta^{\prime}\left(\frac{\Lambda-\Lambda^{\prime}}{2 U}\right)
\end{aligned}
$$

where $k \in[-Q, Q], \Lambda \in[-B, B]$ and $\theta^{\prime}(x)=d \theta / d x(x)$.

\subsection{Ground state}

We consider the "half-filled band" limit, defined as

$$
N-N_{\uparrow \pi}-N_{\downarrow \pi}=L \quad \text { and } \quad 2 N_{\uparrow \bar{\pi}}=N-N_{\uparrow \pi}-N_{\downarrow \pi} .
$$

Using the same arguments as in [6,7], we obtain $Q(\Phi)=\pi$ and $B(\Psi)=\infty$. This can be seen as follows. Taking the normalization relations for $\rho(k)$ and $\sigma(\Lambda)(4.14)$ and inserting there the relations between the counting functions and the densities, we get:

$$
I(Q)-I(-Q)=N-N_{\uparrow \pi}-N_{\downarrow \pi}, \quad J(B)-J(-B)=N_{\uparrow \bar{\pi}}
$$


Inserting the definitions of the counting functions (4.16) in these equations, the following conditions arise: if $Q \rightarrow \pi$ then $N-N_{\uparrow \pi}-N_{\downarrow \pi}=L$ and if $B \rightarrow \infty$ then $2 N_{\uparrow \bar{\pi}}=$ $N-N_{\uparrow \pi}-N_{\downarrow \pi}$.

This limit allows us to find the solution for the densities $\rho(k)$ and $\sigma(\Lambda)$ by Fourier transform:

$$
\begin{aligned}
& \sigma_{0}(\Lambda)=\frac{1}{2 \pi} \int_{0}^{\infty} d w \frac{\cos (w \Lambda)}{\cos (w U)} J_{0}(w), \quad \Lambda \in[-\infty, \infty] \\
& \rho_{0}(k)=\frac{1}{2 \pi}+\frac{\cos k}{\pi} \int_{0}^{\infty} d w \frac{\cos (w \sin k)}{e^{2 U w}+1} J_{0}(w), \quad k \in[-\pi, \pi]
\end{aligned}
$$

with zeroth order Bessel function $J_{0}(x)=\frac{1}{\pi} \int_{0}^{\infty} d w \cos (x \sin w)$.

The ground state energy is then equal:

$$
E=-\mathrm{U}\left(L-2 N_{\bar{\pi}}\right)-2 \sum_{l \in \mathcal{M}_{\bar{\pi} \uparrow} \cup \mathcal{M}_{\bar{\pi} \downarrow}} \cos \left(k_{l}\right)=-\mathrm{U}\left(L-2 N_{\bar{\pi}}\right)-4 L \int_{0}^{\infty} d w \frac{J_{1}(w) J_{0}(w)}{w\left(e^{2 U w}+1\right)}
$$

with order one Bessel function $J_{1}(x)=\frac{x}{\pi} \int_{0}^{\infty} d w \cos (x \sin w) \cos ^{2} w$.

\subsection{String hypothesis}

From the study of the ground state, it is tempting to conjecture that in the thermodynamical limit, the Bethe parameters line up into so-called strings, as for the usual Hubbard model. We remind that the string hypothesis states that all regular solutions $\left\{k_{j}\right\}$ and $\Lambda_{j}$ of Bethe equations (3.7)-(3.11) consist of three kinds of configurations:

1. real $k_{j} \in[-\pi, \pi]$;

2. $\Lambda$ 's combined in $\Lambda$-strings (of arbitrary length $n$ ):

$$
\Lambda_{a, j}^{n}=\Lambda_{a}^{n}+i U(n+1-2 j), \quad j=1, \ldots, n,
$$

3. $2 n k$ 's and $n \Lambda$ 's combined in $k-\Lambda$-strings:

$$
\begin{aligned}
& \Lambda_{a, j}^{\prime n}=\Lambda_{a}^{\prime n}+i U(n+1-2 j), \quad j=1, \ldots, n, \\
& k_{a}^{n, j}=\left\{\begin{array}{lll}
\pi-\arcsin \left(\Lambda_{a}^{\prime n}+i \mathrm{U}(n+1-j)\right), & \text { if } j=1,3, \ldots, 2 n-1 \\
\arcsin \left(\Lambda_{a}^{\prime n}+i \mathrm{U}(n-j)\right), & \text { if } \quad j=2,4, \ldots 2 n-2 \\
\pi-\arcsin \left(\Lambda_{a}^{\prime n}-i n U\right), & \text { if } \quad j=2 n
\end{array}\right.
\end{aligned}
$$

The string centers $\Lambda_{a}^{n}, \Lambda_{a}^{\prime n}$ are real, the parameter $n$ indicates the length of the string and a priori goes from 1 to infinity, the parameter $a$ counts the strings with the same length: $a=1, \ldots, M_{n}, M_{n}$ being the number of strings of length $n$. The branch of $\arcsin (x)$ is chosen between $-\pi / 2$ and $\pi / 2$. 
Using the string hypothesis inside the Bethe equations, especially for equations (4.4) and (4.5), and taking the logarithms, we arrive at the following form of the Bethe Ansatz equations for the (real) centers of the strings, called discrete Takahashi equations:

$$
\begin{aligned}
& k_{j} L=2 \pi I_{j}+2 \pi \Phi-\sum_{n=1}^{\infty}\left(\sum_{a=1}^{M_{n}} \theta\left(\frac{\sin \left(k_{j}\right)-\Lambda_{a}^{n}}{n U}\right)+\sum_{a=1}^{M_{n}^{\prime}} \theta\left(\frac{\sin \left(k_{j}\right)-\Lambda_{a}^{\prime n}}{n U}\right)\right) \\
& 2 L \Re\left(\arcsin \left(\Lambda_{a}^{\prime n}+i n U\right)\right)=2 \pi J_{a}^{\prime n}-2 \pi(\Phi+\Psi)+\sum_{\substack{j=1 \\
j \notin \mathbb{A}}}^{N-2 M^{\prime}} \theta\left(\frac{\Lambda_{a}^{\prime n}-\sin k_{j}}{n U}\right)+ \\
& +\sum_{m=1}^{\infty} \sum_{b=1}^{M_{m}^{\prime}} \Theta_{n m}\left(\frac{\Lambda_{a}^{\prime n}-\Lambda_{b}^{\prime m}}{U}\right) \\
& \sum_{\substack{j=1 \\
j \notin \mathbb{A}}}^{N-2 M^{\prime}} \theta\left(\frac{\Lambda_{a}^{n}-\sin k_{j}}{n U}\right)=2 \pi J_{a}^{n}-2 \pi \Psi+\sum_{m=1}^{\infty} \sum_{b=1}^{M_{m}} \Theta_{n m}\left(\frac{\Lambda_{a}^{n}-\Lambda_{b}^{m}}{U}\right)
\end{aligned}
$$

where $j \in\left[1, N-2 M^{\prime}\right] \backslash \mathbb{A}$ in (4.26) and $M^{\prime}=\sum_{n=1}^{\infty} n M_{n}^{\prime}$. The cut-off for the logarithm is taken as in section 4.1. The function $\Theta_{n m}(x)$ is defined as:

$$
\Theta_{n m}(x)=\sum_{l=\left|\frac{m-n}{2}\right|}^{\frac{m+n}{2}-1}\left(\theta\left(\frac{x}{2 l}\right) \delta_{l \neq 0}+\theta\left(\frac{x}{2(l+1)}\right)\right)
$$

where $\delta_{l \neq 0}$ is 0 when $l$ is 0 , and 1 otherwise.

Looking at the range of the functions entering the BAEs, we can give bounds for the integers $I_{j}, J_{a}^{n}, J_{a}^{\prime n}$ :

$$
\begin{aligned}
& -\frac{L}{2}+\frac{1}{2} \sum_{m=1}^{\infty}\left(M_{m}+M_{m}^{\prime}\right)-\min (\Phi) \leq I_{j} \leq \frac{L}{2}-\frac{1}{2} \sum_{m=1}^{\infty}\left(M_{m}+M_{m}^{\prime}\right)-\max (\Phi) \\
& J_{\min }^{\prime n} \leq J_{a}^{\prime n} \leq J_{\max }^{\prime n} \text { with } \\
& J_{\min }^{\prime n}=-\frac{1}{2}\left(L-N+2 M^{\prime}+N_{\uparrow \pi}+N_{\downarrow \pi}-1-\sum_{m=1}^{\infty} M_{m}^{\prime} t_{n m}\right)+\max (\Phi)+\max (\Psi) \\
& J_{\max }^{\prime n}=\frac{1}{2}\left(L-N+2 M^{\prime}+N_{\uparrow \pi}+N_{\downarrow \pi}-1-\sum_{m=1}^{\infty} M_{m}^{\prime} t_{n m}\right)+\min (\Phi)+\min (\Psi) \\
& J_{\min }^{n} \leq J_{a}^{n} \leq J_{\max }^{n} \text { with } \\
& J_{\min }^{n}=-\frac{1}{2}\left(N-2 M^{\prime}-N_{\uparrow \pi}-N_{\downarrow \pi}-1-\sum_{m=1}^{\infty} M_{m}^{\prime} t_{n m}\right)+\max (\Psi) \\
& J_{\max }^{n}=\frac{1}{2}\left(N-2 M^{\prime}-N_{\uparrow \pi}-N_{\downarrow \pi}-1-\sum_{m=1}^{\infty} M_{m}^{\prime} t_{n m}\right)+\min (\Psi)
\end{aligned}
$$

with $t_{n m}=2 \min (n, m)-\delta_{n m}, \min (A)$ (resp. $\left.\max (A)\right)$ is the minimal value of $\mathrm{A}$ and $I_{j}, J_{a}^{n}, J_{a}^{\prime n}$ are such that: $I_{j}$ is integer (half-integer) if $\sum_{m=1}^{\infty}\left(M_{m}+M_{m}^{\prime}\right)$ is even (odd); $J_{a}^{\prime n}$ 
is integer (half-integer) if $L-\left(N-2 M^{\prime}-N_{\uparrow \pi}-N_{\downarrow \pi}\right)+M_{n}^{\prime}$ is odd (even) and $J_{a}^{n}$ is integer (half-integer) if $\left(N-2 M^{\prime}-N_{\uparrow \pi}-N_{\downarrow \pi}\right)-\sum_{m=1}^{\infty} M_{m} t_{m n}$ is odd (even).

The minimum and maximum of $\Phi$ and $\Psi$ are:

$$
\begin{aligned}
\max (\Phi)= & \frac{N_{\downarrow \bar{\pi}}+N_{\downarrow \mathfrak{m}}-1}{2}-\frac{N_{\downarrow \pi}+1}{L-N_{\downarrow \bar{\pi}}-N_{\downarrow \mathfrak{m}}}+N_{\downarrow \bar{\pi}}+\frac{N_{\downarrow \bar{\pi}}-N_{\downarrow(\mathfrak{m}-1)}\left(N_{\downarrow(\mathfrak{m}-1)}+1\right) / 2}{N_{\downarrow \bar{\pi}}+N_{\downarrow \mathfrak{m}}} \\
\min (\Phi)= & \frac{N_{\downarrow \bar{\pi}}+N_{\downarrow \mathfrak{m}}-1}{2}-\frac{N_{\downarrow \pi}}{L-N_{\downarrow \bar{\pi}}-N_{\downarrow \mathfrak{m}}}-N_{\downarrow \bar{\pi}} \\
& +\frac{\left(N_{\downarrow \bar{\pi}}-N_{\downarrow \mathfrak{m}-1}\right)\left(N_{\downarrow \bar{\pi}}-N_{\downarrow}(\mathfrak{m}-1)\right.}{2\left(N_{\downarrow \bar{\pi}}+N_{\downarrow \mathfrak{m}}\right)}+N_{\downarrow \bar{\pi}}\left(N_{\downarrow \bar{\pi}}+1\right) \\
\max (\Psi)= & \frac{N_{\uparrow \bar{\pi}}+N_{\downarrow \bar{\pi}}+N_{\downarrow \mathfrak{m}}}{2}+\left(N_{\uparrow \pi}+N_{\downarrow \pi}+N_{\downarrow \bar{\pi}}+\left(N_{\uparrow \bar{\pi}}-N_{\uparrow \mathfrak{n}}\right)\right)+\frac{N_{\uparrow \pi}}{L-N_{\uparrow \bar{\pi}}} \\
& -\frac{N_{\downarrow \pi}\left(N_{\downarrow \pi}-1\right)+1}{L-N_{\downarrow \bar{\pi}}-N_{\downarrow \mathfrak{m}}}-\frac{\left(N_{\downarrow \bar{\pi}}-N_{\downarrow \mathfrak{m}-1}\right)\left(N_{\downarrow \bar{\pi}}-N_{\downarrow \mathfrak{m}-1}+1\right)-N_{\downarrow \bar{\pi}}\left(N_{\downarrow \bar{\pi}}-1\right)}{2\left(N_{\downarrow \bar{\pi}}+N_{\downarrow \mathfrak{m}}\right)} \\
& -\frac{\left(N_{\uparrow \bar{\pi}}-N_{\uparrow \mathfrak{n} \mathfrak{n}}\right)\left(N_{\uparrow \bar{\pi}}-N_{\uparrow \mathfrak{n}}-1\right)}{2 N_{\uparrow \bar{\pi}}} \\
\min (\Psi)= & \frac{N_{\uparrow \bar{\pi}}+N_{\downarrow \bar{\pi}}+N_{\downarrow \mathfrak{m}}}{2}+\frac{N_{\uparrow \pi}^{2}+1}{L-N_{\uparrow \bar{\pi}}}-\frac{N_{\downarrow \mathfrak{m}-1}\left(N_{\downarrow \mathfrak{m}-1}+1\right)}{2\left(N_{\downarrow \bar{\pi}}+N_{\downarrow \mathfrak{m}}\right)} \\
& +\frac{\left(N_{\uparrow \bar{\pi}}-N_{\uparrow \mathfrak{n} \mathfrak{n}}\right)\left(N_{\uparrow \bar{\pi}}-N_{\uparrow \mathfrak{n}}+1\right)}{2 N_{\uparrow \bar{\pi}}}
\end{aligned}
$$

If the string hypothesis is still valid, these equations should be the ones to consider for the study of excited states above the ground state described in section 4.3. The first numerical studies we have performed seems to indicate that when the phases $\Psi$ and $\Phi$ are not zero, the string hypothesis needs to be modified: the spaces between the imaginary parts of the Bethe roots seem not to be integers anymore. However, more detailed numerical studies are needed to confirm it. If this alteration is confirmed, than the above Bethe equations will be valid only in the "zero phase subsector".

\section{Applications}

\subsection{Multi-leg Hubbard models}

In this section we consider a particular $\left(\begin{array}{c}1 \mid 2 \\ 1 \mid 0\end{array} ; 1 \mid 0\right)$ Hubbard model. The Hamiltonian (2.13) for superalgebras as introduced in [17] can be written as

$$
H=-\sum_{x=1}^{L}\left((\Sigma P)_{\uparrow x, x+1}+(\Sigma P)_{\downarrow x, x+1}-\frac{U}{4} C_{\uparrow x} C_{\downarrow x}\right), \quad \bmod L
$$

with the gradation introduced in kinetic terms:

$$
(\Sigma P)_{\alpha x, x+1}=\sum_{\bar{\jmath}=2,3}\left\{(-1)^{[\bar{\jmath}]} E_{\alpha x}^{1 \bar{\jmath}} E_{\alpha x+1}^{\bar{\jmath} 1}+E_{\alpha x}^{\bar{\jmath} 1} E_{\alpha x+1}^{1 \bar{\jmath}}\right\}, \quad \text { with } \alpha=\uparrow, \downarrow
$$


We use the Jordan-Wigner transformation (see [16] or [18] for more details):

$$
\begin{array}{ll}
E_{a \uparrow}^{21}=\left(1-n_{a \uparrow}^{c}\right) d_{a \uparrow}^{\dagger} Z_{1 \downarrow}^{L}, & E_{a \downarrow}^{21}=\left(1-n_{a \downarrow}^{c}\right) d_{a \downarrow}^{\dagger} \\
E_{a \uparrow}^{31}=-\left(1-n_{a \uparrow}^{d}\right) c_{a \uparrow}^{\dagger} Z_{1 \downarrow}^{L}, & E_{a \downarrow}^{31}=-\left(1-n_{a \downarrow}^{d}\right) c_{a \downarrow}^{\dagger}
\end{array}
$$

with $n_{a \sigma}^{c}=c_{a \sigma}^{\dagger} c_{a \sigma}, n_{a \sigma}^{d}=d_{a \sigma}^{\dagger} d_{a \sigma}$ and $Z_{1 \downarrow}^{L}=\prod_{i=1}^{L}\left(1-2 n_{i \downarrow}^{c}\right)$.

The Hamiltonian (5.1) finally can be presented in the following form:

$$
\begin{aligned}
H & =H^{H u b}\left(c, c^{\dagger}, U\right)+H^{H u b}\left(d, d^{\dagger}, U\right)+V_{\mathrm{int}}\left(c, c^{\dagger}, d, d^{\dagger}, U\right) \\
H^{H u b}\left(c, c^{\dagger}, U\right) & =-\sum_{i=1}^{L} \sum_{\sigma=\uparrow, \downarrow}\left(c_{k \sigma}^{\dagger} c_{k+1 \sigma}+c_{k+1 \sigma}^{\dagger} c_{k \sigma}\right)+U \sum_{i=1}^{L}\left(1-2 n_{k \uparrow}^{c}\right)\left(1-2 n_{k \downarrow}^{c}\right)
\end{aligned}
$$

with

$$
\begin{aligned}
V_{\mathrm{int}}\left(c, c^{\dagger}, d, d^{\dagger}, U\right)= & -\sum_{i=1}^{L} \sum_{\sigma=\uparrow, \downarrow}\left(\left(c_{k \sigma}^{\dagger} c_{k+1 \sigma}+c_{k+1 \sigma}^{\dagger} c_{k \sigma}\right)\left(n_{k, \sigma}^{d} n_{k+1, \sigma}^{d}-n_{k, \sigma}^{d}-n_{k+1, \sigma}^{d}\right)\right. \\
& \left.+\left(d_{k \sigma}^{\dagger} d_{k+1 \sigma}+d_{k+1 \sigma}^{\dagger} d_{k \sigma}\right)\left(n_{k, \sigma}^{c} n_{k+1, \sigma}^{c}-n_{k, \sigma}^{c}-n_{k+1, \sigma}^{c}\right)\right) \\
& +\frac{U}{4} \sum_{k=1}^{L}\left(\left(1-2 n_{k \uparrow}^{c}\right)\left(1-2 n_{k \uparrow}^{d}\right)+\left(1-2 n_{k \uparrow}^{d}\right)\left(1-2 n_{k \uparrow}^{c}\right)\right. \\
& -2\left(1-n_{k \uparrow}^{c}-n_{k \uparrow}^{d}\right)\left(1+2 n_{k \downarrow}^{c} n_{k \downarrow}^{d}\right)-2\left(1+2 n_{k \uparrow}^{c} n_{k \uparrow}^{d}\right)\left(1-n_{k \downarrow}^{c}-n_{k \downarrow}^{d}\right) \\
& \left.+\left(1+2 n_{k \uparrow}^{c} n_{k \uparrow}^{d}\right)\left(1+2 n_{k \downarrow}^{c} n_{k \downarrow}^{d}\right)\right)
\end{aligned}
$$

The Hamiltonian (5.4) can be interpreted as two periodic lines on which the electrons (described by $c, c^{\dagger}$ for the first line, and by $d, d^{\dagger}$ for the second one) interact via the usual Hubbard Hamitonian (5.5), plus a term of interaction $V_{\text {int }}$ between the two lines. Thus,

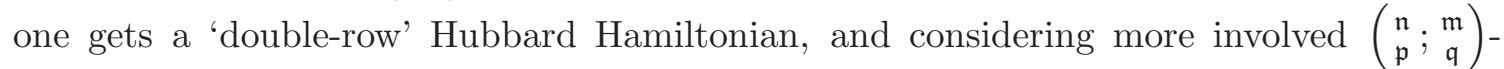
models, one can construct multileg-Hubbards models. In this way, we construct an almost two-dimensional Hubbard model that is still integrable.

The eigenfunctions for this Hamiltonian are made of creator operators $c_{\uparrow}^{\dagger}, c_{\downarrow}^{\dagger}, d_{\uparrow}^{\dagger}$ and $d_{\downarrow}^{\dagger}$. They can be written in the following form and correspond to the solutions constructed in appendix A:

$$
\begin{aligned}
& \Phi_{N_{1}, N_{2}, N_{3}, N_{4}}=\sum_{\vec{x}^{(1)}} \sum_{\vec{x}^{(2)}} \sum_{\vec{x}^{(3)}} \sum_{\vec{x}^{(4)}} \quad \Psi^{\prime}\left(\vec{x}^{(1)}, \vec{x}^{(2)}, \vec{x}^{(3)}, \vec{x}^{(4)}\right) \\
& x_{k}^{(1)} \neq x_{l}^{(1)} \quad x_{k}^{(2)} \neq x_{l}^{(2)}, x_{l}^{(1)} \quad x_{i}^{(3)} \neq x_{j}^{(3)} \quad x_{i}^{(4)} \neq x_{j}^{(4)}, x_{l}^{(3)} \\
& \prod_{j=1}^{N_{1}} c_{x_{j}^{(1)} \uparrow}^{\dagger} \prod_{j=1}^{N_{2}} c_{x_{j}^{(2)} \downarrow}^{\dagger} \prod_{j=1}^{N_{3}} d_{x_{j}^{\dagger}}^{\dagger} \uparrow \prod_{j=1}^{N_{4}} d_{x_{j}^{(4)} \downarrow}^{\dagger} \mid 0>=(-1)^{N_{1}+N_{2}} \phi[\bar{A}] \\
& \text { and } \bar{A}=(\overbrace{3 \uparrow, \ldots, 3 \uparrow}^{N_{1}}, \overbrace{3 \downarrow, \ldots, 3 \downarrow}^{N_{2}}, \overbrace{2 \uparrow, \ldots, 2 \uparrow}^{N_{2}} \overbrace{2 \downarrow, \ldots, 2 \downarrow}^{N_{3}}) \text {. }
\end{aligned}
$$

where $\phi[\bar{A}]$ is the eigenfunction given in (A.1). The particles $c_{\uparrow}^{\dagger}, c_{\downarrow}^{\dagger}, d_{\uparrow}^{\dagger}$ and $d_{\downarrow}^{\dagger}$ correspond to $3 \uparrow, 3 \downarrow, 2 \uparrow$ and $2 \downarrow$ respectively. The particles $c_{\sigma}^{\dagger}$ and $d_{\sigma}^{\dagger}$ can be identified with a (spin up and down) electrons. 
The energy of the excited state $\Phi_{N_{1}, N_{2}, N_{3}, N_{4}}$ reads

$$
E=U(L-2 N)-2 \sum_{l=1}^{N} \cos k_{l} \text { with } N=N_{1}+N_{2}+N_{3}+N_{4}
$$

where the parameters $k_{l}$ are Bethe roots defined by equations:

$$
\begin{aligned}
e^{i k_{j} L} & =\prod_{l=1}^{N_{1}+N_{3}} \frac{\lambda_{l}-\sin k_{j}-i U}{\lambda_{l}-\sin k_{j}+i U} \prod_{i=1}^{N_{4}} b_{i}, \quad \text { for } \quad j \in[1, N] \\
b_{i}^{N_{2}+N_{4}} & =1, \quad \arg \left(b_{i}\right)<\arg \left(b_{i+1}\right), \quad \text { with } \quad i \in\left[1, N_{4}\right], \\
\prod_{j=1}^{N} \frac{\lambda_{l}-\sin k_{j}-i U}{\lambda_{l}-\sin k_{j}+i U} & =e^{\frac{2 \pi i}{N_{1}+N_{3}} \sum_{i=1}^{N_{3}} \bar{n}_{i}} \prod_{i=1}^{N_{4}} b_{i} \prod_{\substack{m=1 \\
m \neq l}}^{N_{1}+N_{3}} \frac{\lambda_{l}-\lambda_{m}-2 i U}{\lambda_{l}-\lambda_{m}+2 i U},
\end{aligned}
$$

with $1 \leq \bar{n}_{1}<\ldots<\bar{n}_{N_{3}} \leq N_{1}+N_{3}$, for $l \in\left[1, N_{1}+N_{3}\right]$.

\subsection{Comparison with AdS/CFT}

The (energy) spectrum of the (usual) Hubbard model has been shown [15] to reproduce correctly the spectrum of the dilation operator in the $s u(2)$ sub-sector up to three loops. The perturbation theory is done at $g \rightarrow 0$ on the SYM-side, while it is done at $\mathfrak{u} \rightarrow \infty$ on the Hubbard's side. Starting at fourth loop, the corrections [19] differ with the Hubbard model. The string Bethe equations (for the $s u(2)$ subsector) read

$$
\left(\frac{x\left(u_{k}+\frac{i}{2}\right)}{x\left(u_{k}-\frac{i}{2}\right)}\right)^{L}=\prod_{\substack{j=1 \\
j \neq k}}^{M} \frac{u_{k}-u_{j}+i}{u_{k}-u_{j}-i} e^{i \theta\left(u_{k}, u_{j}\right)} \quad \text { with } \quad\left\{\begin{array}{l}
u_{j}=\frac{1}{2} \operatorname{cotan}\left(\frac{1}{2} p_{j}\right) \\
x(u)=\frac{u}{2}\left(1+\sqrt{1-\frac{2 g^{2}}{u^{2}}}\right)
\end{array}\right.
$$

where $\theta\left(u_{k}, u_{j}\right)$ is the so-called dressing phase that takes care of the discrepency to the Hubbard model. In general, this phase has two different origins. One is just related to the asymptotic Bethe ansatz and the scattering matrix of the model, and the other cause comes from the so-called wrapping problem, occuring when the order of the expansion in $g$ is bigger than the length of the chain, $L$. When considering the $s u(2)$ sector and the Hubbard model, the wrapping problem has been already 'solved': the Hamiltonian is local, and it is the expansion in term of the coupling constant that makes appear different spin chain models with long range interactions. Hence, for this sector, it is only the dressing factor that has to be implemented.

In the Bethe equation (3.11), such a phase occurs, that depends on some Bethe parameters. Unfortunately, for the present models, the Bethe parameters involved in the phase seem not to be of the type $u_{j}$. However, we believe that the present construction could be a first step for the construction of an integrable model possessing such a dependence. In particular, since the models we construct can be interpreted as multi-leg Hubbard models (see section 5.1), they are free from any wrapping problem. In the context of SYM theories, one should stick to the single circle interpretation, with electrons possessing some internal degrees of freedom (as it is done in the Bethe ansatz of appendix A). Then, to get a true 
$s u(2)$ model, one would have to integrate over the internal degrees of freedom, to get an effective model.

To be more appealing, we reformulate the Bethe equations (5.9)-(5.11) with the notation and half-filling constraint:

$$
2 U=\frac{1}{g \sqrt{2}} ; \quad \frac{\lambda_{\ell}}{2 U}=u_{\ell} ; \quad N=N_{1}+N_{2}+N_{3}+N_{4}=L ; \quad N_{1}+N_{3}=M
$$

Forgetting for a while the state multiplicity, eq. (5.10), we also set

$$
\prod_{i=1}^{N_{4}} b_{i}=e^{\frac{2 i \pi}{L-M} \theta_{24}} \quad \text { and } \quad e^{\frac{2 \pi i}{N_{1}+N_{3}} \sum_{i=1}^{N_{3}} \bar{n}_{i}}=e^{\frac{2 i \pi}{M} \theta_{13}}
$$

Then, we get:

$$
\begin{array}{r}
e^{i k_{j} L}=e^{\frac{2 i \pi}{L-M} \theta_{24}} \prod_{\ell=1}^{M} \frac{u_{\ell}-g \sqrt{2} \sin k_{j}-\frac{i}{2}}{u_{\ell}-g \sqrt{2} \sin k_{j}+\frac{i}{2}} \quad \text { for } \quad j=1, \ldots, L \\
\prod_{j=1}^{L} \frac{u_{l}-g \sqrt{2} \sin k_{j}-\frac{i}{2}}{u_{l}-g \sqrt{2} \sin k_{j}+\frac{i}{2}}=e^{\frac{2 i \pi}{M} \theta_{13}+\frac{2 i \pi}{L-M} \theta_{24}} \prod_{\substack{m=1 \\
m \neq l}}^{M} \frac{u_{l}-u_{m}-i}{u_{l}-u_{m}+i} \quad \text { for } \quad l=1, \ldots, M
\end{array}
$$

The first equation can be used to determine the the Bethe roots $k_{j}$, while the second one has to be compared with (5.12).

\section{Conclusion}

We have presented the Bethe equations for generalized Hubbard models, using the coordinate Bethe ansatz. They look similar to the usual Hubbard model ones, but a phase that appears in the equations. This phase has to be compared to the one appearing in the string Bethe equations. Unfortunately, the present phase is constant (more precisely depends only on the non dynamical parameters associated to the $\bar{\pi} \downarrow$ particles,), while the one appearing in the string context depends on the Bethe parameters (associated here to the $\bar{\pi} \uparrow$ particles). We believe our construction can be used as a first step for constructing an integrable model that would reproduce the expected phase. An open problem is thus to look for an amendment of the construction to provide $k$-dependent phases for AdS/CFT. In that respect, let us note the studies done in [23] that provides an alternative way to get generalized Hubbard models.

The thermodynamical limit has been also discussed and a string hypothesis presented. In some cases (zero phase subsectors), it seems clear that the string hypothesis is correct and similar to the one of the (usual) Hubbard model. However, when the phases are not zero there are presently not enough data to conclude on the validity of the string hypothesis. Thus, we think that more numerical studies are needed to confirm or modify the string hypothesis in the thermodynamical limit.

Finally, we think that our models can be used in condensed matter physics to define of multi-leg Hubbard models. Contrarily to the real two-dimensionnal Hubbard model, these multi-leg Hubbard models are still integrable. Hence, they can be used to get more insight on the two-dimensionnal model using integrable methods. 


\section{A Coordinate Bethe ansatz}

The derivation of the Bethe Ansatz equations of the model is based on the use of the coordinate Bethe Ansatz. The diagonalization of the Hamiltonian (2.13), which involves $\mathfrak{n}+\mathfrak{m}$ types of particles, is done in two main steps. The Hamiltonian eigenfunctions are written as linear combinations of plane waves, whose coefficients are found to be solutions of a new eigenvalue problem, the Hamiltonian of which shows up as a chain of $S$-matrices. This is the first auxiliary problem.

In order to diagonalize this auxiliary Hamiltonian, the corresponding wave function is decomposed as excitations above a suitably chosen pseudo-vacuum with some coefficients. Using a recursive representation of the auxiliary Hamiltonian, recursive relations between these coefficients can be found. The resolution of these relations depend on the effective structure of the Bethe roots, since the choice of the pseudo-vacuum breaks the symmetry between spin up and spin down particles. The different cases are then considered.

\section{A.1 Coordinate Bethe ansatz, level one}

The states corresponding to $N$ excitations are written as

$$
\phi[\bar{A}]=\sum_{\mathbf{x} \in[1, L]} \Psi[\mathbf{x}, \bar{A}] \prod_{i=1}^{N} e_{x_{i}}^{A_{i}} \phi_{0}
$$

with $\bar{A}=(\overbrace{2 \uparrow, \ldots, 2 \uparrow}^{N_{\uparrow 2}}, \ldots, \overbrace{\mathfrak{n} \uparrow, \ldots, \mathfrak{n} \uparrow}^{N_{\uparrow \mathfrak{n}}}, \overbrace{2 \downarrow, \ldots, 2 \downarrow}^{N_{\downarrow 2}}, \ldots, \overbrace{\mathfrak{m} \downarrow, \ldots, \mathfrak{m} \downarrow}^{N_{\downarrow \mathfrak{m}}})$. The sum is done on $\mathbf{x}=\left(x_{1}, x_{2}, \ldots, x_{N}\right)$ without points where two particles with the same spin coincide. The vacuum is chosen as

$$
\phi_{0}=\prod_{k=1}^{L} e_{k}^{1 \uparrow} e_{k}^{1 \downarrow} .
$$

Acting with the Hamiltonian (2.13) on the excited state, we get the eigenvalue equation for the $\Psi$ function:

$$
\begin{aligned}
\sum_{l \in \mathcal{M}_{\bar{\pi} \uparrow \cup \mathcal{M}_{\bar{\pi} \downarrow}}}\left(\Psi\left[\mathbf{x}-\mathbf{e}_{l}+\delta_{1}^{-} \mathbf{e}_{\pi}, \bar{A}\right] \Delta_{l}^{-}+\Psi\left[\mathbf{x}+\mathbf{e}_{l}-\delta_{1}^{+} \mathbf{e}_{\pi}, \bar{A}\right] \Delta_{l}^{+}\right)+ \\
\left(\frac{\mathfrak{u}}{4}\left(L-2 N_{\bar{\pi}}\right)+\mathfrak{u} \sum_{l \in \mathcal{M}_{\bar{\pi}_{\uparrow}}} \sum_{l \in \mathcal{M}_{\bar{\pi}_{\downarrow}}} \delta\left(x_{l}=x_{n}\right)-E\right) \Psi[\mathbf{x}, \bar{A}] \Delta^{3}=0
\end{aligned}
$$

where $\mathbf{e}_{m}$ is an elementary vector in $\mathbb{C}^{N}$ with 1 on the $m$ position and 0 elsewhere. The notation $\Psi\left[\mathbf{x}-\mathbf{e}_{l}+\delta_{1}^{ \pm} \mathbf{e}_{\pi}\right]$ means that, if under the Hamiltonian's action some $\bar{\pi}$-particle (at position $x_{l}$ with some spin $\alpha_{l}$ ) is moved to a place already occupied by some other $\pi$-particle with same spin, then they exchange their places. The symbols $\Delta_{l}^{ \pm}, \Delta^{3}$ represent the exclusion principle for $\bar{\pi}$-particles: they are equal to zero if two such particles with the 
same spin coincide on the same site. Explicitly, they read:

$$
\begin{aligned}
\Delta_{m}^{ \pm} & =\prod_{l \neq m} \prod_{n \neq m} \delta^{\uparrow}\left(x_{l} \neq x_{n}\right) \prod_{l} \delta^{\uparrow}\left(x_{l} \neq x_{m}\right) \delta^{\uparrow}\left(x_{l} \neq x_{m} \pm 1\right), \\
\Delta^{3} & =\prod_{l \neq n} \delta^{\uparrow}\left(x_{l} \neq x_{n}\right),
\end{aligned}
$$

where

$$
\delta^{\downarrow}\left(x_{l} \neq x_{n}\right)=1-\delta\left(x_{l}-x_{n}\right) \quad \text { for } \quad l, n \in \mathcal{M}_{\bar{\pi} \uparrow} \text { or } \mathcal{M}_{\bar{\pi} \downarrow} .
$$

We assume the Bethe hypothesis for the general solution of $\Psi[\mathbf{x}, \bar{A}]$. Dividing the coordinate space $\left(x_{1}, x_{2}, \ldots, x_{N}\right)$ into $N$ ! sectors, we write for $x_{Q(1)}<x_{Q(2)}<\ldots<x_{Q(N)}$,

$$
\Psi_{Q}^{P_{\pi \bar{\pi}}}[\mathbf{x}, \bar{A}]=\sum_{P^{\prime}=P_{\pi} P_{\bar{\pi}}} \Phi_{\bar{A}}\left(\hat{P} Q, \hat{P}^{-1}\right) e^{i \hat{P} \mathbf{k x}}, \quad \hat{P}=P_{\pi \bar{\pi}} P^{\prime}
$$

where $k_{1}, k_{2}, \ldots, k_{N}$ are unequal numbers (the Bethe roots), $Q=\left[q_{1}, q_{2}, \ldots, q_{N}\right]$ is an element of the permutation group $\mathfrak{S}_{N}$ and $\hat{P} \mathbf{k x}=\sum_{i} k_{\hat{P}(i)} x_{i}$. We consider the permutation $\hat{P}$ in its factorized form $\hat{P}=P_{\pi \bar{\pi}} P_{\pi} P_{\bar{\pi}}$, where $P_{\pi \bar{\pi}}$ is a global and fixed permutation of $\pi$ and $\bar{\pi}$ particles, while the terms $P_{\pi}$ and $P_{\bar{\pi}}$ permute $\pi$ and $\bar{\pi}$ particles separately.

The energy depends on the "global" $P_{\pi \bar{\pi}}$ permutation:

$$
E^{P_{\pi \bar{\pi}}}=2 \sum_{l \in \mathcal{M}_{\bar{\pi} \uparrow} \cup \mathcal{M}_{\bar{\pi} \downarrow}} \cos \left(k_{P_{\pi \bar{\pi}}(l)}\right)+\frac{\mathfrak{u}}{4}\left(L-2 N_{\bar{\pi}}\right) .
$$

The coefficients $\Phi_{\bar{A}}\left(\hat{P} Q, \hat{P}^{-1}\right)$ in (A.7) are not all independent. Indeed, using the symmetry of the wave function and the application of the Hamiltonian represented in (A.3), it is possible to reduce their number in several cases:

1. For identical particles of any kind $(\pi$ or $\bar{\pi})$, the wave function satisfies the following symmetry property

$$
\Psi_{Q}^{P_{\pi \bar{\pi}}}[\mathbf{x}, \bar{A}]=\Psi_{Q \Pi_{i i+1}}^{P_{\pi \bar{\pi}}}\left[\Pi_{Q(i) Q(i+1)} \mathbf{x}, \Pi_{Q(i) Q(i+1)} \bar{A}\right]
$$

that leads to the relation

$$
\Phi_{\bar{A}}\left(\hat{P} Q, \hat{P}^{-1}\right)=\Phi_{\bar{A}}\left(\hat{P} Q,\left(\hat{P} \Pi_{Q(i) Q(i+1)}\right)^{-1}\right)
$$

where $\Pi_{a b}$ is the permutation of objects $a$ and $b$, the indices $Q(i), Q(i+1)$ correspond to identical particles.

2. For particles with the same spin we also impose some kind of weak "exclusion principle" such that the wave function vanishes if two particles with the same spin coincide (but the particles can differ by their $\pi$ or $\bar{\pi}$ type). This principle is verified for $\pi$-particles if we use (A.3) with two particles at positions $x_{Q(i)}=x_{Q(i+1)}-1$ (in the sector $x_{Q(1)} \ll \ldots \ll$ $x_{Q(i)}<x_{Q(i+1)} \ll x_{Q(N)}$, the notation $\ll$ means that the particles are far enough from each other). We generalize this condition to any kind of particles. It leads to:

$$
\Psi_{Q}^{P_{\pi \bar{\pi}}}[\mathbf{x}, \bar{A}]_{x_{Q(i)}=x_{Q(i+1)}}=0, \quad A_{Q(i)}, A_{Q(i+1)} \in \mathcal{N}_{\uparrow} \cup \mathcal{N}_{\downarrow} \text { or } \overline{\mathcal{N}}_{\uparrow} \cup \overline{\mathcal{N}}_{\downarrow}
$$


that is to say

$$
\Phi_{\bar{A}}\left(\hat{P} Q, \hat{P}^{-1}\right)=-\Phi_{\bar{A}}\left(\hat{P} Q \Pi_{i i+1},\left(\hat{P} \Pi_{Q(i) Q(i+1)}\right)^{-1}\right)
$$

for any $i$ such that $A_{Q(i)}, A_{Q(i+1)} \in \mathcal{N}_{\uparrow} \cup \mathcal{N}_{\downarrow}$ or $\overline{\mathcal{N}}_{\uparrow} \cup \overline{\mathcal{N}}_{\downarrow}$.

3. For particles with different spins, there is no exclusion principle and they can be at the same site. Thus, let us consider the case: $x_{Q(1)} \ll \ldots \ll x_{Q(i)}=x_{Q(i+1)} \ll x_{Q(N)}$ and ask for the continuity of the wave function on the boundary of the two domains $Q$ and $Q \Pi_{i i+1}$ :

$$
\Psi_{Q}^{P_{\pi \bar{\pi}}}[\mathbf{x}, \bar{A}]_{x_{Q(i)}=x_{Q(i+1)}}=\Psi_{Q \Pi_{i i+1}}^{P_{\pi \bar{\pi}}}[\mathbf{x}, \bar{A}]_{x_{Q(i)}=x_{Q(i+1)}} .
$$

For two $\bar{\pi}$-particles, it implies the relation, for $A_{Q(i)} \in \overline{\mathcal{N}}_{\uparrow(\downarrow)}$ and $A_{Q(i+1)} \in \overline{\mathcal{N}}_{\downarrow(\uparrow)}$,

$$
\begin{aligned}
\Phi_{\bar{A}}\left(\hat{P} Q, \hat{P}^{-1}\right)+\Phi_{\bar{A}}\left(\hat{P} Q \Pi_{i i+1},\left(\hat{P} \Pi_{Q(i) Q(i+1)}\right)^{-1}\right)= \\
\Phi_{\bar{A}}\left(\hat{P} Q,\left(\hat{P} \Pi_{Q(i) Q(i+1)}\right)^{-1}\right)+\Phi_{\bar{A}}\left(\hat{P} Q \Pi_{i i+1}, \hat{P}^{-1}\right)
\end{aligned}
$$

while for two $\pi$-particles it leads, for $A_{Q(i)} \in \mathcal{N}_{\uparrow(\downarrow)}$ and $A_{Q(i+1)} \in \mathcal{N}_{\downarrow(\uparrow)}$, to

$$
\Phi_{\bar{A}}\left(\hat{P} Q \Pi_{i i+1}, \hat{P}^{-1}\right)=\Phi_{\bar{A}}\left(\hat{P} Q, \hat{P}^{-1}\right) .
$$

When one particle is of type $\pi$ and the other one of type $\bar{\pi}$, we find, for $A_{Q(i)} \in \mathcal{N}_{\uparrow(\downarrow)}$ and $A_{Q(i+1)} \in \overline{\mathcal{N}}_{\downarrow(\uparrow)}$,

$$
\Phi_{\bar{A}}\left(\hat{P} Q \Pi_{i i+1}, \hat{P}^{-1}\right)=\Phi_{\bar{A}}\left(\hat{P} Q, \hat{P}^{-1}\right) .
$$

There is another condition for $\bar{\pi}$-particles when using (A.3). This is the usual relation on the coefficient $\Phi_{\bar{A}}\left(\hat{P} Q, \hat{P}^{-1}\right)$ obtained in the $\left(\begin{array}{l}2 \\ 1\end{array} ; \begin{array}{l}2 \\ 1\end{array}\right)$ Hubbard model for spin up and spin down interacting electrons:

$$
\begin{array}{r}
\sum_{\substack{m \neq Q_{i}, Q_{i+1} \\
P_{Q}}}\left(\Psi_{Q}^{P_{\pi \bar{\pi}}}\left[\mathbf{x}-\mathbf{e}_{m}, \bar{A}\right]+\Psi_{Q}^{P_{\pi \bar{\pi}}}\left[\mathbf{x}+\mathbf{e}_{m}, \bar{A}\right]\right)+\left(\frac{\mathfrak{u}}{4}\left(L-2 N_{\bar{\pi}}\right)+\mathfrak{u}-E\right) \Psi_{Q}^{P_{\pi \bar{\pi}}}[\mathbf{x}, \bar{A}]+ \\
\Psi_{Q}^{P_{\bar{\pi}}}\left[\mathbf{x}-\mathbf{e}_{Q_{i}}, \bar{A}\right]+\Psi_{Q \Pi_{i i+1}}^{P_{\pi \bar{\pi}}}\left[\mathbf{x}+\mathbf{e}_{Q_{i}}, \bar{A}\right]+\Psi_{Q \Pi_{i i+1}}^{P_{\pi \bar{\pi}}}\left[\mathbf{x}-\mathbf{e}_{Q_{i+1}}, \bar{A}\right]+\Psi_{Q}^{P_{\pi \bar{\pi}}}\left[\mathbf{x}+\mathbf{e}_{Q_{i+1}}, \bar{A}\right]=0
\end{array}
$$

Skipping the intermediate calculations and combining the results with (A.14), we can write the conditions on $\Phi\left(\hat{P} Q, \hat{P}^{-1}\right)$ in a matrix form:

$$
\left(\begin{array}{c}
\Phi\left(\Pi_{a b} \hat{P} Q, \hat{P}^{-1}\right) \\
\Phi\left(\Pi_{a b} \hat{P} Q,\left(\Pi_{a b} \hat{P}\right)^{-1}\right)
\end{array}\right)=\left(\begin{array}{cc}
t_{a b} & r_{a b} \\
r_{a b} & t_{a b}
\end{array}\right)\left(\begin{array}{c}
\Phi\left(\hat{P} Q, \hat{P}^{-1}\right) \\
\Phi\left(\hat{P} Q,\left(\Pi_{a b} \hat{P}\right)^{-1}\right)
\end{array}\right)
$$

with $a=\hat{P} Q(i), b=\hat{P} Q(i+1)$ and

$$
t_{a b}=\frac{2 i\left(\lambda_{a}-\lambda_{b}\right)}{\mathfrak{u}+2 i\left(\lambda_{a}-\lambda_{b}\right)}, \quad r_{a b}=\frac{-\mathfrak{u}}{\mathfrak{u}+2 i\left(\lambda_{a}-\lambda_{b}\right)}, \quad \lambda_{a}=\sin k_{a}
$$

These equations hold for any type of $\bar{\pi}$-excitations (any value of $A_{Q_{i}}, A_{Q_{i+1}}$ being $\bar{\pi}$ particles). 
4. Finally we consider the interaction between $\pi$ and $\bar{\pi}$ particles with the same spin. Let $A_{Q(i)}$ be a $\bar{\pi}$-particle, $A_{Q(i+1)}$ be a $\pi$-particle, with coordinates $x_{Q(i)}=x_{Q(i+1)}-1$, thus $\delta_{1}^{+}=1$ and $\delta_{1}^{-}=0$. Using the equation (A.3) we derive the relation:

$$
\Psi_{Q \Pi_{i i+1}}^{P_{\pi \bar{\pi}}}\left[\mathbf{x}+\mathbf{e}_{Q(i)}-\mathbf{e}_{Q(i+1)}, \bar{A}\right]-\Psi_{Q}^{P_{\pi \bar{\pi}}}\left[\mathbf{x}+\mathbf{e}_{Q(i)}, \bar{A}\right]=0
$$

that implies the following condition on $\Phi_{\bar{A}}\left(\hat{P} Q, \hat{P}^{-1}\right)$ :

$$
\Phi_{\bar{A}}\left(\Pi_{\hat{P} Q(i) \hat{P} Q(i+1)} \hat{P} Q, \hat{P}^{-1}\right)=e^{i k_{\hat{P} Q(i+1)}} \Phi_{\bar{A}}\left(\hat{P} Q, \hat{P}^{-1}\right) .
$$

We rewrite now all obtained conditions (A.10), (A.12)-(A.17) and (A.20) in a more compact form. Let us introduce, for $P^{\prime} \equiv \hat{P} Q \in \mathfrak{S}_{N}$ and $Q^{\prime} \equiv \hat{P}^{-1} \in \mathfrak{S}_{N}$,

$$
\hat{\Phi}\left(P^{\prime}\right) \equiv \sum_{Q^{\prime}, \bar{A}} \Phi_{\bar{A}}\left(P^{\prime}, Q^{\prime}\right) \prod_{i=1}^{N} e_{i}^{A_{Q^{\prime}(i)}}
$$

where the summation is over all types of excitations and all corresponding sectors. The vector $\prod_{i=1}^{N} e_{i}^{A_{Q^{\prime}(i)}}$ belongs to $V_{1} \otimes \ldots \otimes V_{N}$, where $V$ is spanned by $\left\{e^{2 \uparrow}, e^{3 \uparrow}, \ldots, e^{\mathfrak{n} \uparrow}, e^{2 \downarrow}, e^{3 \downarrow}, \ldots, e^{\mathfrak{m} \downarrow}\right\}$ and represents one type of $N$ excitations. The ordering of the particles is chosen such that, for $Q^{\prime}=i d$, the vector $\prod_{i=1}^{N} e_{i}^{A_{i}}$ is taken as in (A.1):

$$
\prod_{i=1}^{N} e_{i}^{A_{i}}=\overbrace{e^{2 \uparrow} \otimes \ldots \otimes e^{2 \uparrow}}^{N_{\uparrow 2}} \otimes \ldots \otimes \overbrace{e^{\mathfrak{n} \uparrow} \otimes \ldots \otimes e^{\mathfrak{n} \uparrow}}^{N_{\uparrow \mathfrak{n}}} \otimes \overbrace{e^{2 \downarrow} \otimes \ldots \otimes e^{2 \downarrow}}^{N_{\downarrow}} \otimes \ldots \otimes \overbrace{e^{\mathfrak{m} \downarrow} \otimes \ldots \otimes e^{\mathfrak{m} \downarrow}}^{N_{\downarrow \mathfrak{m}}}
$$

To clarify the notation for $\hat{\Phi}\left(P^{\prime}\right)$, we give as an example the case $N=2$ :

$$
\begin{aligned}
\hat{\Phi}\left(P^{\prime}\right)= & \sum_{j=2}^{\mathfrak{n}} \Phi_{(j \uparrow, j \uparrow)}\left(P^{\prime}, i d\right) e_{1}^{j \uparrow} e_{2}^{j \uparrow}+\sum_{j=2}^{\mathfrak{m}} \Phi_{(j \downarrow, j \downarrow)}\left(P^{\prime}, i d\right) e_{1}^{j \downarrow} e_{2}^{j \downarrow} \\
& +\sum_{2 \leq j<k \leq \mathfrak{n}}\left(\Phi_{(j \uparrow, k \uparrow)}\left(P^{\prime}, i d\right) e_{1}^{j \uparrow} e_{2}^{k \uparrow}+\Phi_{(j \uparrow, k \uparrow)}\left(P^{\prime}, \Pi_{12}\right) e_{1}^{k \uparrow} e_{2}^{j \uparrow}\right) \\
& +\sum_{2 \leq j<k \leq \mathfrak{m}}\left(\Phi_{(j \downarrow, k \downarrow)}\left(P^{\prime}, i d\right) e_{1}^{j \downarrow} e_{2}^{k \downarrow}+\Phi_{(j \downarrow, k \downarrow)}\left(P^{\prime}, \Pi_{12}\right) e_{1}^{k \downarrow} e_{2}^{j \downarrow}\right) \\
& +\sum_{j=2}^{\mathfrak{n}} \sum_{k=2}^{\mathfrak{m}}\left(\Phi_{(j \uparrow, k \downarrow)}\left(P^{\prime}, i d\right) e_{1}^{j \uparrow} e_{2}^{k \downarrow}+\Phi_{(j \uparrow, k \downarrow)}\left(P^{\prime}, \Pi_{12}\right) e_{1}^{k \downarrow} e_{2}^{j \uparrow}\right)
\end{aligned}
$$

Then, all the relations can be expressed in a matrix form. For $N=2$, it reads

$$
\hat{\Phi}\left(\Pi_{12} P\right)=S_{12}^{(1)}\left(k_{1}, k_{2}\right) \hat{\Phi}(P)
$$

where $S_{12}^{(1)}\left(k_{1}, k_{2}\right)$ (simply denoted by $S_{12}^{(1)}$ in the following) acts on elementary vectors $e_{1}^{A_{1}} e_{2}^{A_{2}}$ as

1) for $\pi$-particles:

$$
\begin{aligned}
& S_{12}^{(1)} e_{1}^{A_{1}} e_{2}^{A_{2}}=-e_{1}^{A_{2}} e_{2}^{A_{1}}, \text { for } A_{1}, A_{2} \in \mathcal{N}_{\uparrow(\downarrow)} \\
& S_{12}^{(1)} e_{1}^{A_{1}} e_{2}^{A_{2}}=e_{1}^{A_{1}} e_{2}^{A_{2}}, \text { for } A_{1} \in \mathcal{N}_{\uparrow(\downarrow)}, A_{2} \in \mathcal{N}_{\downarrow}(\uparrow)
\end{aligned}
$$


2) for $\bar{\pi}$-particles:

$$
\begin{aligned}
& S_{12}^{(1)} e_{1}^{\bar{A}_{1}} e_{2}^{\bar{A}_{2}}=-e_{1}^{\bar{A}_{2}} e_{2}^{\bar{A}_{1}}, \text { for } \bar{A}_{1}, \bar{A}_{2} \in \overline{\mathcal{N}}_{\uparrow(\downarrow)} \\
& S_{12}^{(1)} e_{1}^{\bar{A}_{1}} e_{2}^{\bar{A}_{2}}=t_{12} e_{1}^{\bar{A}_{1}} e_{2}^{\bar{A}_{2}}+r_{12} e_{1}^{\bar{A}_{2}} e_{2}^{\bar{A}_{1}}, \text { for } \bar{A}_{1} \in \overline{\mathcal{N}}_{\uparrow(\downarrow)}, \bar{A}_{2} \in \overline{\mathcal{N}}_{\downarrow(\uparrow)}
\end{aligned}
$$

3) for mixed $\pi$ and $\bar{\pi}$-particles:

$$
\begin{aligned}
& S_{12}^{(1)} e_{1}^{A_{1}} e_{2}^{\bar{A}_{2}}=e^{-i k_{1}} e_{1}^{A_{1}} e_{2}^{\bar{A}_{2}} \text { and } S_{12}^{(1)} e_{1}^{\bar{A}_{2}} e_{2}^{A_{1}}=e^{i k_{2}} e_{1}^{\bar{A}_{2}} e_{2}^{A_{1}}, \text { for } A_{1} \in \mathcal{N}_{\uparrow(\downarrow)}, \bar{A}_{2} \in \overline{\mathcal{N}}_{\uparrow(\downarrow)} \\
& S_{12}^{(1)} e_{1}^{A_{1}} e_{2}^{\bar{A}_{2}}=e_{1}^{A_{1}} e_{2}^{\bar{A}_{2}} \text { and } S_{12}^{(1)} e_{1}^{\bar{A}_{2}} e_{2}^{A_{1}}=e_{1}^{\bar{A}_{2}} e_{2}^{A_{1}}, \text { for } A_{1} \in \mathcal{N}_{\uparrow(\downarrow)}, \bar{A}_{2} \in \overline{\mathcal{N}}_{\downarrow(\uparrow)} \quad(\mathrm{A} .27)
\end{aligned}
$$

The parameters $t_{12}, r_{12}$ are defined in (A.18) and $\mathcal{N}_{\uparrow(\downarrow)}, \overline{\mathcal{N}}_{\uparrow(\downarrow)}$ are given in (2.4).

The notation $A \in \mathcal{N}_{\uparrow(\downarrow)}$ means that the excitation $A$ can be equal to any value in $\mathcal{N}_{\uparrow(\downarrow)}$ except the value $1 \uparrow(\downarrow)$ since it was chosen as the vacuum at first level.

For an arbitrary $N$ excitations number, we write

$$
\hat{\Phi}\left(\Pi_{a b} P\right)=S_{a b}^{(1)}\left(k_{a}, k_{b}\right) \hat{\Phi}(P),
$$

where the matrix $S_{a b}^{(1)}\left(k_{a}, k_{b}\right)$ has the same meaning as above but acts nontrivialy only on the $V_{a} \otimes V_{b}$ vector space. It can be written using permutations and projectors:

$$
\begin{aligned}
S_{a b}^{(1)}\left(k_{a}, k_{b}\right)= & -\left(P_{a b}^{\mathcal{N}_{\uparrow}, \mathcal{N}_{\uparrow}}+P_{a b}^{\overline{\mathcal{N}}_{\uparrow}, \overline{\mathcal{N}}_{\uparrow}}+P_{a b}^{\mathcal{N}_{\downarrow}, \mathcal{N}_{\downarrow}}+P_{a b}^{\overline{\mathcal{N}}_{\downarrow}, \overline{\mathcal{N}}_{\downarrow}}\right)+e^{-i k_{a}}\left(I d_{a b}^{\mathcal{N}_{\uparrow}, \overline{\mathcal{N}}_{\uparrow}}+I d_{a b}^{\mathcal{N}_{\downarrow}, \overline{\mathcal{N}}_{\downarrow}}\right)+ \\
& +e^{i k_{b}}\left(I d_{a b}^{\overline{\mathcal{N}}_{\uparrow}, \mathcal{N}_{\uparrow}}+I d_{a b}^{\mathcal{N}_{\downarrow}, \mathcal{N}_{\downarrow}}\right)+I d_{a b}^{\mathcal{N}_{\uparrow}, \mathcal{N}_{\downarrow} \cup \overline{\mathcal{N}}_{\downarrow}}+I d_{a b}^{\mathcal{N}_{\downarrow}, \mathcal{N}_{\uparrow} \cup \overline{\mathcal{N}_{\uparrow}}}+I d_{a b}^{\mathcal{N}_{\uparrow}, \mathcal{N}_{\downarrow}}+I d_{a b}^{\mathcal{N}_{\downarrow}, \mathcal{N}_{\uparrow}}+ \\
& +\left(t_{a b} I d_{a b}^{\overline{\mathcal{N}}_{\uparrow}, \overline{\mathcal{N}}_{\downarrow}}+r_{a b} P_{a b}^{\mathcal{N}_{\uparrow}, \overline{\mathcal{N}}_{\downarrow}}\right)+\left(t_{a b} I d_{a b}^{\overline{\mathcal{N}}_{\downarrow}, \overline{\mathcal{N}}_{\uparrow}}+r_{a b} P_{a b}^{\overline{\mathcal{N}}_{\downarrow}, \overline{\mathcal{N}}_{\uparrow}}\right)
\end{aligned}
$$

where we defined

$$
I d_{a b}^{\mathcal{L}, \mathcal{L}^{\prime}}=\sum_{i \in \mathcal{L}} \sum_{j \in \mathcal{L}^{\prime}} E_{a}^{i i} E_{b}^{j j} \quad \text { and } \quad P_{a b}^{\mathcal{L}, \mathcal{L}^{\prime}}=\sum_{i \in \mathcal{L}} \sum_{j \in \mathcal{L}^{\prime}} E_{a}^{i j} E_{b}^{j i}, \quad \mathcal{L}, \mathcal{L}^{\prime}=\mathcal{N}_{\uparrow}, \mathcal{N}_{\downarrow}, \overline{\mathcal{N}}_{\uparrow}, \overline{\mathcal{N}}_{\downarrow} .
$$

The matrix $S_{a b}^{(1)}\left(k_{a}, k_{b}\right)$ satisfies the Yang-Baxter equation (2.8).

Now we can write the periodic boundary conditions. Let $C_{N}=\Pi_{N 1} \ldots \Pi_{N N-1}$ be a circular permutation. The sites $L+1$ and 1 being identified, we have the periodicity conditions:

$$
\Psi_{Q C_{N}}^{P_{\pi \bar{\pi}}}\left[\mathbf{x}-\mathbf{e}_{Q(N)} L, \bar{A}\right]=\Psi_{Q}^{P_{\pi \bar{\pi}}}[\mathbf{x}, \bar{A}] .
$$

In terms of $\hat{\Phi}(P)$ this yields the condition

$$
\hat{\Phi}(P C)=e^{i k_{P(N)} L} \hat{\Phi}(P) .
$$

If we choose $P=C_{N}^{N-j}$ with $j=1, \ldots, N$, we can derive a system of equations on the coefficients $\hat{\Phi}(i d)$ which is called the "auxiliary problem":

$$
\mathfrak{h}_{j} \hat{\Phi}(i d)=e^{i k_{j} L} \hat{\Phi}(i d) \quad \text { with } \quad \mathfrak{h}_{j}=S_{j+1, j}^{(1)} \ldots S_{N j}^{(1)} S_{1 j}^{(1)} \ldots S_{j-1, j}^{(1)}, \quad j=1, \ldots, N
$$

where we omitted the arguments of the $S$-matrices, $S_{a b}^{(1)} \equiv S_{a b}^{(1)}\left(k_{a}, k_{b}\right)$.

The Yang-Baxter equation for the $S$ matrix implies that $\left[\mathfrak{h}_{j}, \mathfrak{h}_{k}\right]=0$ for all $j, k$, so that the new Hamiltonians $\mathfrak{h}_{j}$ can be simultaneously diagonalized: we do it in the folowing section. 


\section{A.2 Auxiliary problem, level two}

In order to simplify the calculations, we make the change: $S_{12}^{(1)} \rightarrow-S_{12}^{(1)} \equiv S_{12}$. The eigenvalue problem to be solved reads

$$
S_{j+1, j} \ldots S_{N j} S_{1 j} \ldots S_{j-1, j} \phi=\Lambda_{j} \phi .
$$

Again we use the coordinate Bethe ansatz. At this level we have $\mathfrak{n}+\mathfrak{m}-2$ types of different excitations: $e^{2 \uparrow}, \ldots, e^{\mathfrak{n} \uparrow}, e^{2 \downarrow}, \ldots, e^{\mathfrak{m} \downarrow}$. We choose as the reference state (pseudovacuum):

$$
\phi_{0}^{(1)}=\prod_{k=1}^{N} e_{k}^{\mathfrak{m} \downarrow}
$$

The eigenvectors for this auxiliary problem are given by

$$
\begin{aligned}
\phi^{(1)}[\bar{A}] & =\sum_{\mathbf{x} \in[1, N]} \Psi^{(1)}[\mathbf{x}, \bar{A}] \prod_{n=1}^{K} e_{x_{n}}^{A_{n}} \phi_{0}^{(1)} \\
K & =\sum_{i=2}^{\mathfrak{p}} N_{\uparrow i}+\sum_{i=2}^{\mathfrak{q}} N_{\downarrow i}+\sum_{i=\mathfrak{p}+1}^{\mathfrak{n}} N_{\uparrow i}+\sum_{i=\mathfrak{q}+1}^{\mathfrak{m}^{-1}} N_{\downarrow}, \\
\bar{A} & =(\underbrace{2 \uparrow, \ldots, 2 \uparrow}_{N_{\uparrow 2}}, \ldots, \underbrace{\mathfrak{n} \uparrow, \ldots, \mathfrak{n} \uparrow}_{N_{\uparrow \mathfrak{n}}}, \underbrace{2 \downarrow, \ldots, 2 \downarrow}_{N_{\downarrow 2}}, \ldots, \underbrace{(\mathfrak{m}-1) \downarrow, \ldots,(\mathfrak{m}-1) \downarrow}_{N_{\downarrow}(\mathfrak{m}-1)}) .
\end{aligned}
$$

The ansatz for $\Psi^{(1)}[\mathbf{x}, \bar{A}] \equiv \Psi_{Q}[\mathbf{x}, \bar{A}]$ can be written as, for $x_{Q(1)}<x_{Q(2)}<\ldots<x_{Q(K)}$ and $Q \in \mathfrak{S}_{K}$,

$$
\Psi_{Q}[\mathbf{x}, \bar{A}]=\sum_{P} \Phi_{\bar{A}}\left(P Q, P^{-1}\right) \prod_{i \in \mathcal{M}_{\pi \uparrow}} h_{x_{i}}\left(a_{P(i)}\right) \prod_{i \in \mathcal{M}_{\bar{\pi} \uparrow}} f_{x_{i}}\left(\lambda_{P(i)}\right) \prod_{i \in \mathcal{M}_{\pi \downarrow}} \bar{h}_{x_{i}}\left(a_{P(i)}\right) \prod_{i \in \mathcal{M}_{\bar{\pi} \downarrow}} g_{x_{i}}\left(b_{P(i)}\right),
$$

where $P=P_{\pi \uparrow} P_{\bar{\pi} \uparrow} P_{\pi \downarrow} P_{\bar{\pi} \downarrow}$ is a factorized permutation such that $P_{\pi \uparrow}, P_{\bar{\pi} \uparrow}, P_{\pi \downarrow}$ and $P_{\bar{\pi} \downarrow}$ are the sets of permutations in $\mathfrak{S}_{K}$ permuting only particles of type $\pi \uparrow, \bar{\pi} \uparrow, \pi \downarrow$ and $\bar{\pi} \downarrow$ respectively, e.g. $P_{A}(i)=i$ for $i \notin \mathcal{M}_{A}$ and $P_{A}(j) \in \mathcal{M}_{A}$ for $j \in \mathcal{M}_{A}$ with $A=\pi \uparrow, \bar{\pi} \uparrow$, $\pi \downarrow, \bar{\pi} \downarrow$. We recall that the sets $\mathcal{M}_{\pi_{\uparrow}}, \mathcal{M}_{\bar{\pi}_{\uparrow}}, \mathcal{M}_{\pi_{\downarrow}}$ and $\mathcal{M}_{\bar{\pi}_{\downarrow}}$ are defined in section 3 .

The eigenfunctions $f_{x}(\lambda), g_{x}(b), h_{x}(a)$ and $\bar{h}_{x}(a)$ correspond to one-particle solutions and are defined as

$$
f_{x}(\lambda)=\prod_{m=1}^{x-1}\left(-\frac{i \sin k_{m}+i \lambda+\frac{\mathfrak{u}}{4}}{i \sin k_{m+1}+i \lambda-\frac{\mathfrak{u}}{4}}\right) ; \quad g_{x}(b)=b^{x} ; \quad \bar{h}_{x}(a)=h_{x}(a)=\delta(x-a) .
$$

Although the functions $h$ and $\bar{h}$ are identical, they will be associated to different kinds of particles ( $\pi \uparrow$ and $\pi \downarrow$ respectively). Since they will lead to different Hamiltonian eigenvalues (see below (A.44)), we have chosen to distinguish them in (A.37). The form of the eigenfunctions (A.38) is supported by the fact that the eigenfunction in (A.33) should be independent of the index $j$.

We can remark that the Bethe roots $a_{i}$ for $i \in \mathcal{M}_{\pi_{\downarrow}}$ or $\mathcal{M}_{\pi_{\uparrow}}$ corresponding to $\pi_{\downarrow}$ and $\pi_{\uparrow}$ particles are already quantized on the small chain. In order to have an independent number of eigenvectors (A.36), one should take the following conditions into account:

$$
a_{i}<a_{i+1} \text { for } i \in \mathcal{M}_{\pi_{\uparrow(\downarrow)}}
$$

that leads to the fact that in (A.37), $P_{\pi \uparrow}$ and $P_{\pi \downarrow}$ are fixed: $P_{\pi_{\uparrow(\downarrow)}} Q(j)=$ const (see below). 
The action of the auxiliary Hamiltonian (A.33) on the wavefunction can be calculated using the relations given in the previous section. In the recursive representation of the Hamiltonian (for details, see [24])

$$
S_{j-k, j} \ldots S_{j-1, j} \phi^{(1)}[\bar{A}] \equiv \sum_{\mathbf{x} \in[1, N]} \hat{H}_{j}^{(k)} \Psi_{Q}[\mathbf{x}, \bar{A}] \prod_{n=1}^{K} e_{x_{n}}^{A_{n}} \phi_{0}^{(1)}
$$

one can derive the following recursive relations between the coefficients $\hat{H}_{j}^{(k)} \Psi_{Q}[\mathbf{x}, \bar{A}]$ for decreasing $k$. Nontrivial relations occur when $\hat{H}_{j}^{(k)}$ acts on $\Psi_{Q}[\mathbf{x}, \bar{A}]$ for which some coordinate $x_{p}$ is equal to $j$ and/or $j-k$. One gets two sets of relations. The first one reads:

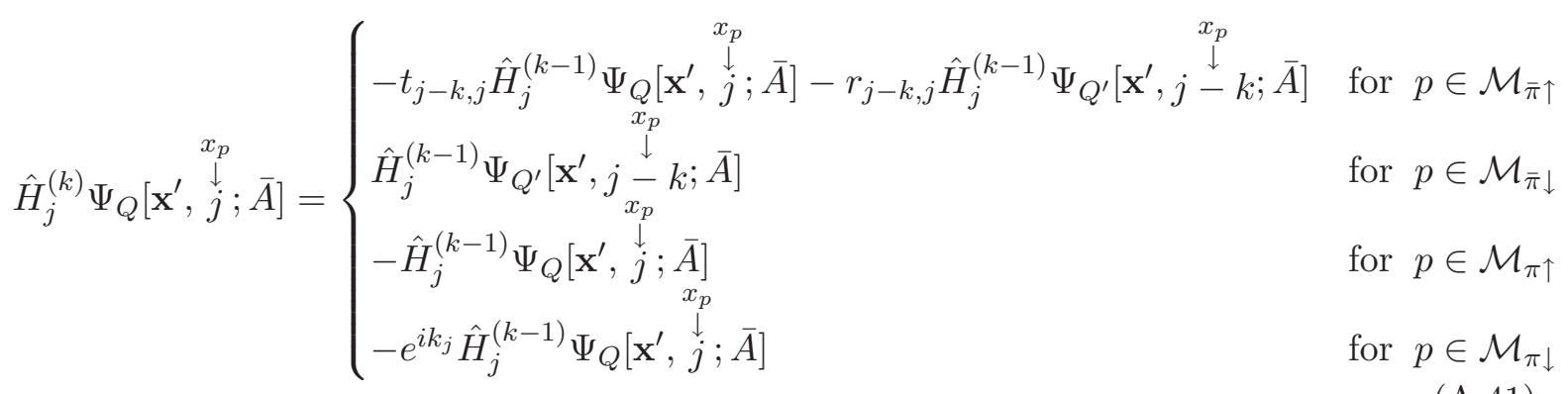

The above relations are invariant if we exchange $j$ with $j-k$ except the last one, that is transformed as follows:

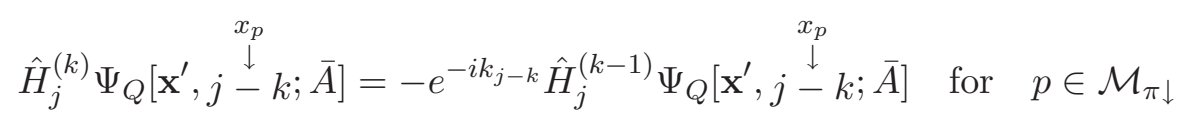

The second set of relations is

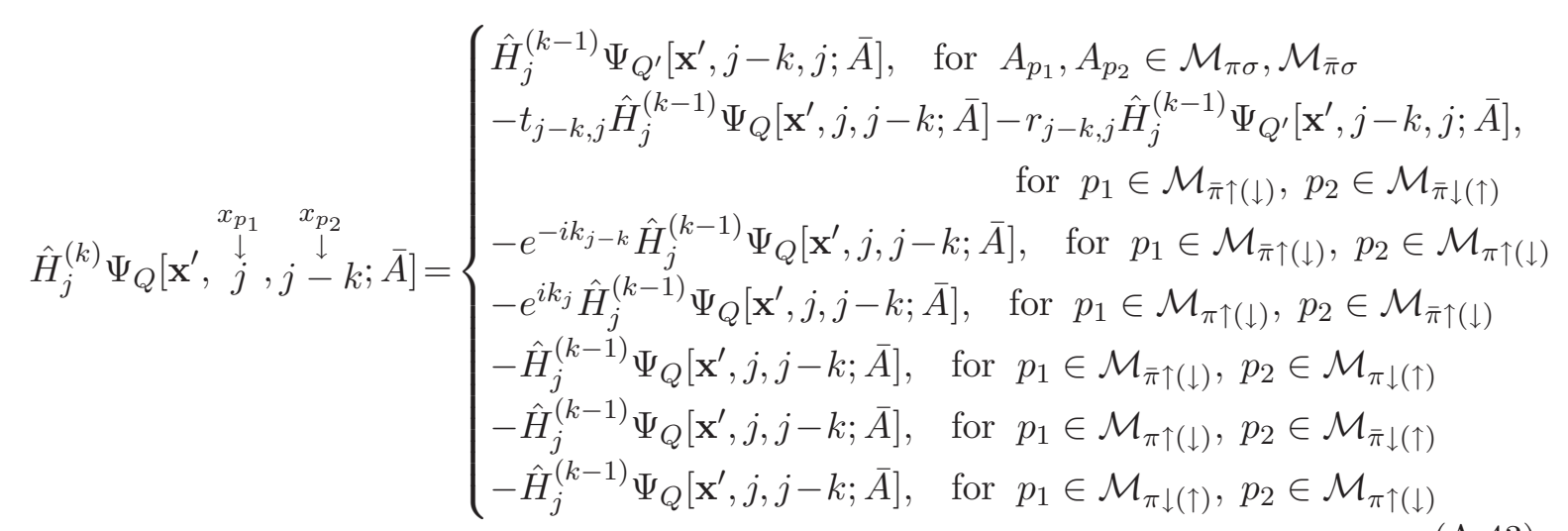

In eqs. (A.41)-(A.43), the vector $\mathbf{x}^{\prime}$ corresponds to the vector $\mathbf{x}$ without the components $x_{p}$ or $x_{p_{1}}, x_{p_{2}}$ and does not contain any position $x_{q}=j$ or $j-k$.

Using these relations, we can then apply the whole product of $S$-matrices (A.34) on a one-excitation function. We get for $x \neq j$

$$
\begin{aligned}
& \hat{H}_{j}^{(N-1)} f_{x}(\lambda)=\sigma_{j}(\lambda) f_{x}(\lambda) \quad \text { and } \quad \hat{H}_{j}^{(N-1)} g_{x}(b)=b g_{x}(b) \\
& \hat{H}_{j}^{(N-1)} h_{x}(a)=-h_{x}(a) \quad \text { and } \quad \hat{H}_{j}^{(N-1)} \bar{h}_{x}(a)=-e^{-i k_{x}} \bar{h}_{x}(a)
\end{aligned}
$$


and for $x=j$

$$
\begin{aligned}
& \hat{H}_{j}^{(N-1)} f_{j}(\lambda)=\prod_{l=1, l \neq j}^{N} \sigma_{l}(\lambda) f_{j}(\lambda) \text { and } \hat{H}_{j}^{(N-1)} g_{j}(b)=b^{1-N} g_{j}(b) \\
& \hat{H}_{j}^{(N-1)} h_{j}(a)=(-1)^{N-1} h_{j}(a) \text { and } \hat{H}_{j}^{(N-1)} \bar{h}_{j}(a)=(-1)^{N-1} e^{i k_{j}(N-1)} \bar{h}_{j}(a)
\end{aligned}
$$

where

$$
\sigma_{j}(\lambda)=-\frac{i \sin \left(k_{j}\right)+i \lambda+\frac{\mathfrak{u}}{4}}{i \sin \left(k_{j}\right)+i \lambda-\frac{\mathfrak{u}}{4}}
$$

Now we will consider the $K$-excitations eigenvector (A.36) with the ansatz (A.37). There are three different possible cases:

I) there exists a Bethe root $a_{l}=j$ for some $l \in \mathcal{M}_{\pi \uparrow}$, with $j$ being the index in (A.33),

II) there exists another Bethe root $a_{m}=j$ for some $m \in \mathcal{M}_{\pi \downarrow}$,

III) there is no such Bethe roots.

We detail the calculations for each of these cases.

I) There exists a Bethe root $a_{l}=j$ for some $l \in \mathcal{M}_{\boldsymbol{\pi} \uparrow}$. In this first case, let us introduce a set of integers $\left\{\alpha_{i} \in[1, K]\right\}_{i \in \mathcal{M}_{\pi \uparrow}}$ such that we have $Q\left(\alpha_{i}\right) \in \mathcal{M}_{\pi \uparrow}$. The set is ordered: $\alpha_{i}<\alpha_{i+1}$. We also define $\alpha_{l}$ such that $x_{Q\left(\alpha_{l}\right)}=j$.

The Hamiltonian acting on the wave function gives the following result:

$$
\begin{aligned}
& \hat{H}_{j}^{(N-1)} \Psi_{Q}\left(\mathbf{x} ; x_{Q\left(\alpha_{1}\right)}, \ldots, x_{Q\left(\alpha_{l-1}\right)}, \quad \stackrel{\substack{x_{Q\left(\alpha_{l}\right)} \\
j}}{j}, x_{Q\left(\alpha_{l+1}\right)}, \ldots, x_{Q\left(\alpha_{N_{\uparrow \pi}}\right)}\right) \\
& =(-1)^{N-1-N_{\uparrow \pi}} e^{i k_{j} N_{\uparrow \pi}} \Psi_{Q C_{\pi \uparrow}}\left(\mathbf{x} ; x_{Q\left(\alpha_{2}\right)}, \ldots, \quad \stackrel{\substack{x_{Q\left(\alpha_{l}\right)} \\
j}}{j}, x_{Q\left(\alpha_{l+1}\right)}, x_{Q\left(\alpha_{l+2}\right)}, \ldots, x_{Q\left(\alpha_{1}\right)}\right) \\
& =\Lambda_{j} \Psi_{Q}\left(\mathbf{x} ; x_{Q\left(\alpha_{1}\right)}, \ldots, x_{Q\left(\alpha_{l-1}\right)}, j, x_{Q\left(\alpha_{l+1}\right)}, \ldots, x_{Q\left(\alpha_{N_{\uparrow \pi}}\right)}\right)
\end{aligned}
$$

and finally we get the condition on $\Phi_{\bar{A}}\left(P Q, P^{-1}\right)$ :

$$
\frac{\Phi_{\bar{A}}\left(P^{\prime} Q C_{\pi \uparrow}, P^{\prime-1}\right)}{\Phi_{\bar{A}}\left(P Q, P^{-1}\right)}=\Lambda_{j}(-1)^{N-1-N_{\uparrow \pi}} e^{-i k_{j} N_{\uparrow \bar{\pi}}} .
$$

In equality (A.48), we have introduced $C_{\pi \uparrow}=\Pi_{\alpha_{N_{\uparrow}}}^{\alpha_{1}} \ldots \Pi_{\alpha_{N_{\uparrow \uparrow}}}^{\alpha_{N_{\uparrow}-1}}$ and for $P=$ $P_{\pi \uparrow} P_{\pi \downarrow} P_{\bar{\pi} \uparrow} P_{\bar{\pi} \downarrow}, P^{\prime}=P_{\pi \uparrow}^{\prime} P_{\pi \downarrow} P_{\bar{\pi} \uparrow} P_{\bar{\pi} \downarrow}$ where $P_{\pi \uparrow}^{\prime}=\Pi_{N_{\uparrow \pi}}^{N_{\uparrow \pi}-1} \ldots \Pi_{N_{\uparrow \pi}}^{1} P_{\pi \uparrow}$.

If we change the sector $Q \rightarrow Q \Pi_{a b}$, which means that we interchange two identical particles $x_{Q(a)}$ and $x_{Q(b)}$, the coefficient $\Phi_{\bar{A}}\left(P Q \Pi_{a b}, P^{-1}\right)$ should remain invariant:

$$
\Phi_{\bar{A}}\left(P^{\prime} Q \Pi_{a b}, P^{\prime-1}\right)=\Phi_{\bar{A}}\left(P Q, P^{-1}\right) \quad \text { with } \quad Q(a), Q(b) \in \mathcal{M}_{\pi \uparrow}
$$

where $P^{\prime}=P_{\pi \uparrow}^{\prime} P_{\pi \downarrow} P_{\bar{\pi} \uparrow} P_{\bar{\pi} \downarrow}$ and $P_{\pi \uparrow}^{\prime}$ is such that $P_{\pi \uparrow}^{\prime}=P_{\pi \uparrow} \Pi_{Q(a) Q(b)}$.

The relation (A.48) can be simplified to:

$$
\frac{\Phi_{\bar{A}}\left(P Q, P^{-1} \tilde{C}_{\pi \uparrow}\right)}{\Phi_{\bar{A}}\left(P Q, P^{-1}\right)}=\Lambda_{j}(-1)^{N-N_{\uparrow \pi}} e^{-i k_{j} N_{\uparrow \bar{\pi}}}
$$


with $\tilde{C}_{\pi \uparrow}=\Pi_{N_{\uparrow \pi}}^{1} \ldots \Pi_{N_{\uparrow \pi}}^{N_{\uparrow \pi}-1}$. Now, taking the product of this equation by itself $N_{\uparrow \pi}$ times and changing $P \rightarrow \tilde{C}_{\pi \uparrow} P \rightarrow \tilde{C}_{\pi \uparrow}^{2} P \rightarrow \ldots \rightarrow \tilde{C}_{\pi \uparrow}^{N_{\uparrow \pi}} P$, we obtain

$$
\Lambda_{j}(-1)^{N-N_{\uparrow \pi}} e^{-i k_{j} N_{\uparrow \bar{\pi}}}=e^{2 \pi i \frac{m_{\uparrow \pi}}{N_{\uparrow \pi}}} \quad\left(m_{\uparrow \pi}=1, \ldots, N_{\uparrow \pi}\right)
$$

It determines the eigenvalue of the auxiliary problem for spin up $\pi$-particles. We also recall that the result has to be multiplied by $(-1)^{N-1}$ in order to come back to the initial problem due to the sign change done at the beginning of the section, see (A.34).

II) There exists another Bethe root $a_{m}=j$ for some $m \in \mathcal{M}_{\pi \downarrow}$. Following the same steps as in the first case, we obtain

$$
\frac{\Phi_{\bar{A}}\left(P Q, P^{-1} \tilde{C}_{\pi \downarrow}\right)}{\Phi_{\bar{A}}\left(P Q, P^{-1}\right)}=\Lambda_{j}(-1)^{N-N_{\downarrow \pi}} e^{-i k_{j}\left(N_{\downarrow \bar{\pi}}+N_{\downarrow \mathfrak{m}}\right)}
$$

where $P=P_{\pi \uparrow} P_{\pi \downarrow} P_{\bar{\pi} \uparrow} P_{\bar{\pi} \downarrow}, P^{\prime}=P_{\pi \uparrow} P_{\pi \downarrow}^{\prime} P_{\bar{\pi} \uparrow} P_{\bar{\pi} \downarrow}$ and $\tilde{C}_{\pi \downarrow}=\Pi_{N_{\downarrow}}^{1} \ldots \Pi_{N_{\downarrow}}^{N_{\downarrow \pi}-1}$.

Finally using the same trick for the product of equations, we obtain

$$
\Lambda_{j}(-1)^{N-N_{\downarrow \pi}} e^{-i k_{j}\left(N_{\downarrow \bar{\pi}}+N_{\downarrow \mathfrak{m}}\right)}=e^{2 \pi i \frac{m_{\downarrow \pi}}{N_{\downarrow \pi}}}, m_{\downarrow \pi}=1, \ldots, N_{\downarrow \pi}
$$

It allows to determine the eigenvalue of the auxiliary problem for $\pi \downarrow$ particles. Again, this result should be multiplied by a factor $(-1)^{N-1}$ to come back to the initial problem.

For identical particles we have the relation:

$$
\Phi_{\bar{A}}\left(P^{\prime} Q \Pi_{a b}, P^{\prime-1}\right)=\Phi_{\bar{A}}\left(P Q, P^{-1}\right) \text {, with } Q(a), Q(b) \in \mathcal{M}_{\pi \uparrow}
$$

where $P^{\prime}=P_{\pi \uparrow} P_{\pi \downarrow}^{\prime} P_{\bar{\pi} \uparrow} P_{\bar{\pi} \downarrow}$ and $P_{\pi \downarrow}^{\prime}$ is such that $P_{\pi \downarrow}^{\prime}=P_{\pi \downarrow} \Pi_{Q(a) Q(b)}$.

III) There is no such Bethe root. In this last case, we have $\pi$-particles with spin up or down distributed anywhere on the chain but the site $j$. Acting with the Hamiltonian (A.33) on the wavefunction (A.37) we get

$$
\hat{H}_{j}^{(N-1)} \Psi_{Q}[\mathbf{x}, \bar{A}]=(-1)^{N_{\pi \uparrow}+N_{\pi \downarrow}} \prod_{l \in \mathcal{M}_{\pi \downarrow}} e^{-i k_{a_{l}}} \prod_{i \in \mathcal{M}_{\bar{\pi} \uparrow}} \sigma_{j}\left(\lambda_{i}\right) \prod_{i \in \mathcal{M}_{\bar{\pi} \downarrow}} b_{i} \Psi_{Q}[\mathbf{x}, \bar{A}], \quad \text { if } \mathbf{x} \neq j
$$

with the following conditions on the coefficients $\Phi_{\bar{A}}\left(P Q, P^{-1}\right)$ :

1) for all $P Q(i), P Q(i+1)$ in $\mathcal{M}_{\bar{\pi} \uparrow}$

$$
\frac{\Phi_{\bar{A}}\left(P Q \Pi_{i i+1},\left(P \Pi_{Q(i) Q(i+1)}\right)^{-1}\right)}{\Phi_{\bar{A}}\left(P Q, P^{-1}\right)}=\frac{i \lambda_{P Q(i)}-i \lambda_{P Q(i+1)}-\frac{\mathfrak{u}}{2}}{i \lambda_{P Q(i)}-i \lambda_{P Q(i+1)}+\frac{\mathfrak{u}}{2}} \equiv \alpha_{P Q(i) P Q(i+1)}^{-1}
$$

2) for all $P Q(i), P Q(i+1)$ in $\mathcal{M}_{\bar{\pi} \downarrow}$ we impose

$$
\frac{\Phi_{\bar{A}}\left(P Q \Pi_{i i+1},\left(P \Pi_{Q(i) Q(i+1)}\right)^{-1}\right)}{\Phi_{\bar{A}}\left(P Q, P^{-1}\right)}=1
$$


3) for all $P Q(i) \in \mathcal{M}_{\bar{\pi} \downarrow}$ and $P Q(i+1) \in \mathcal{M}_{\bar{\pi} \uparrow}$

$$
\frac{\Phi_{\bar{A}}\left(P Q \Pi_{i i+1}, P^{-1}\right)}{\Phi_{\bar{A}}\left(P Q, P^{-1}\right)}=b_{P Q(i)}^{-1}
$$

the same relation holds for all $P Q(i) \in \mathcal{M}_{\bar{\pi} \uparrow}, P Q(i+1) \in \mathcal{M}_{\bar{\pi} \downarrow}$ and changing $Q \rightarrow Q \Pi_{i i+1}$.

4) for all $P Q(i) \in \mathcal{M}_{\pi \uparrow}$ and $P Q(i+1) \in \mathcal{M}_{\bar{\pi} \uparrow}$

$$
\frac{\Phi_{\bar{A}}\left(P Q \prod_{i i+1}, P^{-1}\right)}{\Phi_{\bar{A}}\left(P Q, P^{-1}\right)}=e^{-i k_{a_{P Q(i)}}} \sigma_{a_{P Q(i)}}\left(\lambda_{P Q(i+1)}\right)
$$

5) for all $P Q(i) \in \mathcal{M}_{\pi \downarrow}$ and $P Q(i+1) \in \mathcal{M}_{\bar{\pi} \uparrow}$

$$
\frac{\Phi_{\bar{A}}\left(P Q \Pi_{i i+1}, P^{-1}\right)}{\Phi_{\bar{A}}\left(P Q, P^{-1}\right)}=e^{i k_{a_{P Q(i)}}} \sigma_{a_{P Q(i)}}\left(\lambda_{P Q(i+1)}\right)
$$

6) for all $P Q(i) \in \mathcal{M}_{\pi \uparrow}$ and $P Q(i+1) \in \mathcal{M}_{\bar{\pi} \downarrow}$

$$
\frac{\Phi_{\bar{A}}\left(P Q \Pi_{i i+1}, P^{-1}\right)}{\Phi_{\bar{A}}\left(P Q, P^{-1}\right)}=b_{P Q(i+1)}
$$

7) for all $P Q(i) \in \mathcal{M}_{\pi \downarrow}$ and $P Q(i+1) \in \mathcal{M}_{\bar{\pi} \downarrow}$

$$
\frac{\Phi_{\bar{A}}\left(P Q \Pi_{i i+1}, P^{-1}\right)}{\Phi_{\bar{A}}\left(P Q, P^{-1}\right)}=b_{P Q(i+1)}
$$

In addition, for all $P Q(i), P Q(i+1)$ in $\mathcal{M}_{\bar{\pi} \uparrow}$ or $\mathcal{M}_{\bar{\pi} \downarrow}$ we impose the condition for identical particles:

$$
\frac{\Phi_{\bar{A}}\left(P Q,\left(P \Pi_{Q(i) Q(i+1)}\right)^{-1}\right)}{\Phi_{\bar{A}}\left(P Q, P^{-1}\right)}=1
$$

For the perodicity conditions, there are again two different subcases:

III-A) a $\bar{\pi} \downarrow$-particle is on the $j$ site,

III-B) a $\bar{\pi} \uparrow$-particle is on the $j$ site.

Each case leading to slightly different conditions, we treat them separately.

III-A) A $\overline{\boldsymbol{\pi}} \downarrow$-particle is on the $\boldsymbol{j}$ site. The periodic boundary condition on the coefficients $\Phi_{\bar{A}}\left(P Q, P^{-1}\right)$ comes from the relations

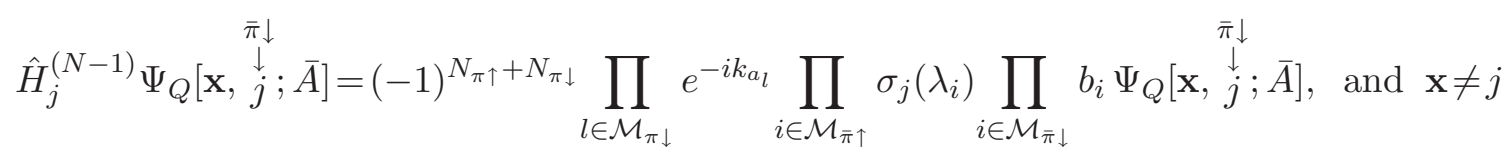

Using relations (A.56) to (A.63) we can calculate $\hat{H}_{j}^{(N-1)} \Psi_{Q}[\mathbf{x}, j ; \bar{A}]$ to get the condition

$$
\frac{\Phi_{\bar{A}}\left(P Q C_{K}, P^{-1}\right)}{\Phi_{\bar{A}}\left(P Q, P^{-1}\right)}=\left[b_{P Q(K)}\right]^{N} \quad \text { with } \quad C_{K}=\Pi_{K}^{1} \ldots \Pi_{K}^{K-1} .
$$


We start with $Q=Q_{\overline{\bar{\pi}} \downarrow}$ such that

$$
Q_{\bar{\pi} \downarrow}(i)=\left\{\begin{array}{ll}
i, & \text { for } i \notin \mathcal{M}_{\bar{\pi} \downarrow} \\
j \in \mathcal{M}_{\bar{\pi} \downarrow}, & \text { for } i \in \mathcal{M}_{g}
\end{array} \Rightarrow P Q(i)= \begin{cases}m \equiv \hat{P}(i) \in[1, K] \cap \mathcal{M}_{\bar{\pi} \downarrow}, & i \notin \mathcal{M}_{\bar{\pi} \downarrow} \\
P_{\bar{\pi} \downarrow} Q_{\bar{\pi} \downarrow}(i) \equiv \tilde{P}(i) \in \mathcal{M}_{\bar{\pi} \downarrow}, & i \in \mathcal{M}_{\bar{\pi} \downarrow}\end{cases}\right.
$$

and

$$
P Q C_{K}=\Pi_{\tilde{P}(K)}^{\hat{P}(1)} \Pi_{\tilde{P}(K)}^{\hat{P}(2)} \ldots \Pi_{\tilde{P}(K)}^{\hat{P}\left(K-N_{\downarrow \bar{\pi}}\right)} \Pi_{\tilde{P}(K)}^{\tilde{P}\left(K-N_{\downarrow \bar{\pi}}+1\right)} \ldots \Pi_{\tilde{P}(K)}^{\tilde{P}(K-1)} P Q
$$

Using relations (A.61), (A.62) and "conjugated" (A.58) when $Q \rightarrow Q \Pi_{i i+1}$, the coefficient $\Phi_{\bar{A}}\left(P Q C_{K}, P^{-1}\right)$ simplifies to:

$$
\begin{aligned}
\Phi_{\bar{A}}\left(P Q C_{K}, P^{-1}\right) & =\Phi_{\bar{A}}\left(\Pi_{\tilde{P}(K)}^{\hat{P}(1)} \Pi_{\tilde{P}(K)}^{\hat{P}(2)} \ldots \Pi_{\tilde{P}(K)}^{\hat{P}\left(K-N_{\downarrow \bar{\pi}}\right)} \Pi_{\tilde{P}(K)}^{\tilde{P}\left(K-N_{\downarrow \bar{\pi}}+1\right)} \ldots \Pi_{\tilde{P}(K)}^{\tilde{P}(K-1)} P Q, P^{-1}\right) \\
& =\left[b_{\tilde{P}(K)}\right]^{N_{\uparrow \pi}+N_{\uparrow \bar{\pi}}+N_{\downarrow \pi}} \Phi_{\bar{A}}\left(\Pi_{\tilde{P}(K)}^{\tilde{P}\left(K-N_{\downarrow \bar{\pi}}+1\right)} \ldots \Pi_{\tilde{P}(K)}^{\tilde{P}(K-1)} P Q, P^{-1}\right) .
\end{aligned}
$$

Finally we have the equation

$$
\Phi_{\bar{A}}\left(\Pi_{\tilde{P}(K)}^{\tilde{P}\left(K-N_{\downarrow \bar{\pi}}+1\right)} \ldots \Pi_{\tilde{P}(K)}^{\tilde{P}(K-1)} P Q, P^{-1}\right)=\left[b_{\tilde{P}(K)}\right]^{N-\left(N_{\uparrow \pi}+N_{\uparrow \bar{\pi}}+N_{\downarrow \pi}\right)} \Phi_{\bar{A}}\left(P Q, P^{-1}\right)
$$

In the next step of the procedure, we introduce the vector $\hat{\Phi}_{\bar{\pi} \downarrow}(P)$ :

$$
\hat{\Phi}_{\bar{\pi} \downarrow}(P) \equiv \sum_{\bar{A} \in G} \sum_{Q^{\prime} \in \mathfrak{S}_{N_{\downarrow} \bar{\pi}}} \Phi_{\bar{A}}\left(P, Q^{\prime}\right) \prod_{i \in \mathcal{M}_{\bar{\pi} \downarrow}} e_{i}^{\bar{A}_{Q^{\prime}(i)}}
$$

where $G=\operatorname{span}\{(\mathfrak{q}+1) \downarrow, \ldots,(\mathfrak{m}-1) \downarrow\}$. The particle order is chosen such that for $Q^{\prime}=i d$, the vector $\prod_{i \in \mathcal{M}_{\bar{\pi} \downarrow}} e_{i}^{\bar{A}_{i}}$ is equal to $\overbrace{e^{(\mathfrak{q}+1) \downarrow} \otimes \ldots \otimes e^{(\mathfrak{q}+1) \downarrow}}^{N_{\downarrow}(\mathfrak{q}+1)} \otimes \ldots \otimes \overbrace{e^{(\mathfrak{m}-1) \downarrow} \otimes \ldots \otimes e^{(\mathfrak{m}-1) \downarrow}}^{N_{\downarrow}(\mathfrak{m}-1)}$. The permutation $Q^{\prime}$ is the image of $Q_{\bar{\pi} \downarrow}$ when one shifts by $K-N_{\downarrow \bar{\pi}}$ the space of integers on which the permutation group $\mathfrak{S}_{N_{\downarrow} \bar{\pi}}$ acts.

The relations (A.57) and (A.63) can be gathered in $\hat{\Phi}_{\bar{\pi} \downarrow}(P)$ :

$$
\hat{\Phi}_{\bar{\pi} \downarrow}\left(\Pi_{a b} P\right)=\mathcal{P}_{a b} \hat{\Phi}_{\bar{\pi} \downarrow}(P)
$$

where $\mathcal{P}_{a b}$ is the permutation acting on the particles located at the $a$ and $b$ positions in $\hat{\Phi}_{\bar{\pi} \downarrow}(P)$. The periodicity problem can then be rewritten in the following form

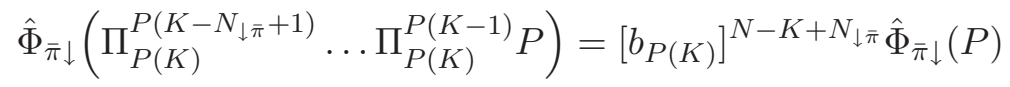

which can be simplified to

$$
\mathcal{P}_{P\left(K-N_{\downarrow}+1\right) P(K)} \cdots \mathcal{P}_{P(K-1) P(K)} \hat{\Phi}_{\bar{\pi} \downarrow}(P)=\left[b_{P(K)}\right]^{N-K+N_{\downarrow \bar{\pi}} \hat{\Phi}_{\bar{\pi} \downarrow}(P)}
$$

Choosing $P=C_{\bar{\pi} \downarrow}^{-m}$ with $C_{\bar{\pi} \downarrow}=\Pi_{K}^{K-N_{\downarrow}+1} \ldots \Pi_{K}^{K-1}$, we get the following Bethe equation

$$
\begin{aligned}
&\left(b_{m}\right)^{N-K+N_{\downarrow \bar{\pi}}}= \exp \left(\frac{2 \pi i}{N_{\downarrow \bar{\pi}}} \sum_{j=1}^{N_{\downarrow \bar{\pi}}-N_{\downarrow(\mathfrak{m}-1)}} n_{j}\right), m \in \mathcal{M}_{\bar{\pi} \downarrow} \\
& \text { with } \quad 1 \leq n_{1}<\ldots<n_{N_{\downarrow \bar{\pi}}-N_{\downarrow}(\mathfrak{m}-1)} \leq N_{\downarrow \bar{\pi}}
\end{aligned}
$$


In order to get all quantum numbers which characterize the eigenfunction and obtain the right number of states, one should solve the permutation problem. We will take the result from [18] (section on permutation problem of $(g l(\mathfrak{n} \mid \mathfrak{m}) \oplus g l(2))$ model). We get additional sets of integers

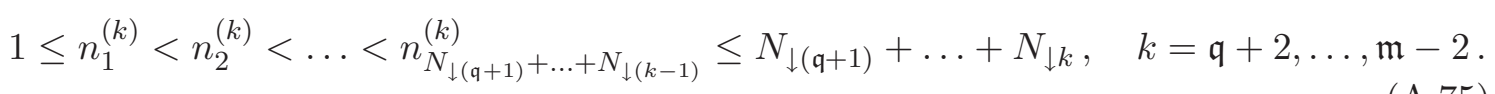

One can remark that if we set $k=\mathfrak{m}-1$ in the above condition, we recover the relation (A.74). This result ends the first subcase.

III-B) A $\overline{\boldsymbol{\pi}} \uparrow$-particle is on the $\boldsymbol{j}$ site. The periodic boundary conditions on the coefficients $\Phi_{\bar{A}}\left(P Q, P^{-1}\right)$ can be written analogously to the previous case

$$
\frac{\Phi_{\bar{A}}\left(P Q C_{K}, P^{-1}\right)}{\Phi_{\bar{A}}\left(P Q, P^{-1}\right)}=\prod_{l=1}^{N} \sigma_{l}\left(\lambda_{P Q(K)}\right) \quad \text { for } \quad Q \quad \text { such that } \quad Q(K) \in \mathcal{M}_{\bar{\pi} \uparrow} .
$$

We proceed as in the case III-A, but instead we choose $Q=Q_{\bar{\pi} \uparrow} C_{K}^{K-N_{\uparrow \pi}-N_{\uparrow \bar{\pi}}}$. Here $Q_{\bar{\pi} \uparrow}$ defined similarly to $Q_{\bar{\pi} \downarrow}: Q_{\bar{\pi} \uparrow}(i) \in \mathcal{M}_{\bar{\pi} \uparrow}$ if $i \in \mathcal{M}_{\bar{\pi} \uparrow}$ and $Q_{\bar{\pi} \uparrow}(i)=i$ for the remaining indices. The cyclic permutation is chosen such that all $\pi$ and $\bar{\pi}$ down particles are moved to the begining from their original ordering for $Q=i d$, that is $C_{K}^{K-N_{\uparrow \pi}-N_{\uparrow \bar{\pi}}}(i)=\left(i+N_{\uparrow \pi}+\right.$ $\left.N_{\uparrow \bar{\pi}}\right) \bmod K$ for any $i$. This choice implies in particular the following relation:

$$
\begin{aligned}
& P Q C_{K}=\left(\prod_{i \in \mathcal{M}_{\pi \downarrow}} \Pi_{\tilde{P}\left(N_{\uparrow \pi}+N_{\uparrow \bar{\pi}}\right)}^{i}\right)\left(\prod_{i \in \mathcal{M}_{\bar{\pi} \downarrow}}^{\longrightarrow} \Pi_{\tilde{P}\left(N_{\uparrow \pi}+N_{\uparrow \bar{\pi}}\right)}^{P_{\bar{\pi}}(i)}\right)\left(\prod_{i \in \mathcal{M}_{\pi \uparrow}}^{\longrightarrow} \Pi_{\tilde{P}\left(N_{\uparrow \pi}+N_{\uparrow \bar{\pi}}\right)}^{i}\right) \times
\end{aligned}
$$

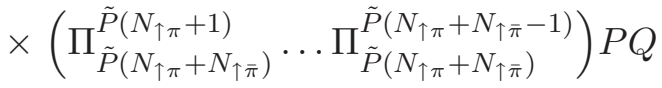

where $\tilde{P}=P_{\bar{\pi} \uparrow} Q_{\bar{\pi} \uparrow}$ and $P Q=P_{\bar{\pi} \downarrow} \tilde{P} C_{K}^{K-N_{\uparrow \pi}-N_{\uparrow \bar{\pi}}}$. Here, due to the choice of $Q, P_{\pi \downarrow}$ and $P_{\pi \uparrow}$ are equal to identity. The symbol $\prod_{i \in \mathcal{M}_{A}} \Pi_{\tilde{P}\left(N_{\uparrow \pi}+N_{\uparrow \bar{\pi}}\right)}^{i}$ denotes the ordered product of permutation: $\ldots \Pi_{\tilde{P}\left(N_{\uparrow \pi}+N_{\uparrow \bar{\pi}}\right)}^{i} \prod_{\tilde{P}\left(N_{\uparrow \pi}+N_{\uparrow \bar{\pi}}\right)}^{i+1} \cdots$

Next, we use relations (A.58), (A.59) and (A.60) to simplify the coefficient $\Phi_{P^{-1}}^{P Q C_{K}}$ :

$$
\begin{aligned}
& \Phi_{\bar{A}}\left(P Q C_{K}, P^{-1}\right)=\prod_{i \in \mathcal{M}_{\pi \downarrow}} e^{i k_{a_{i}}} \sigma_{a_{i}}\left(\lambda_{\tilde{P}\left(N_{\uparrow \pi}+N_{\uparrow \bar{\pi}}\right)}\right) \prod_{i \in \mathcal{M}_{\bar{\pi} \downarrow}} b_{i}^{-1} \prod_{i \in \mathcal{M}_{\pi \uparrow}} e^{-i k_{a_{i}}} \sigma_{a_{i}}\left(\lambda_{\tilde{P}\left(N_{\uparrow \pi}+N_{\uparrow \bar{\pi}}\right)}\right) \times \\
& \times \Phi_{\bar{A}}\left(\Pi_{\tilde{P}\left(N_{\uparrow \pi}+N_{\uparrow \bar{\pi}}\right)}^{\tilde{P}\left(N_{\uparrow \pi}+1\right)} \ldots \Pi_{\tilde{P}\left(N_{\uparrow \pi}+N_{\uparrow \bar{\pi}}\right)}^{\tilde{P}\left(N_{\uparrow \uparrow}+N_{\uparrow \bar{\pi}}-1\right)} P Q, P^{-1}\right)
\end{aligned}
$$

and finally we get

$$
\begin{aligned}
& \prod_{i \in \mathcal{M}_{\pi \downarrow}} e^{i k_{a_{i}}} \sigma_{a_{i}}\left(\lambda_{\tilde{P}\left(N_{\uparrow \pi}+N_{\uparrow \bar{\pi}}\right)}\right) \prod_{i \in \mathcal{M}_{\bar{\pi} \downarrow}} b_{i}^{-1} \prod_{i \in \mathcal{M}_{\pi \uparrow}} e^{-i k_{a_{i}}} \sigma_{a_{i}}\left(\lambda_{\tilde{P}\left(N_{\uparrow \pi}+N_{\uparrow \bar{\pi}}\right)}\right) \times \\
& \Phi_{\bar{A}}\left(\Pi_{\tilde{P}\left(N_{\uparrow \pi}+N_{\uparrow \bar{\pi}}\right)}^{\tilde{P}\left(N_{\uparrow}+1\right)} \ldots \Pi_{\tilde{P}\left(N_{\uparrow \pi}+N_{\uparrow \bar{\pi}}\right)}^{\tilde{P}\left(N_{\uparrow}+N_{\uparrow \bar{\pi}}-1\right)} P Q, P^{-1}\right)=\prod_{l=1}^{N} \sigma_{l}\left(\lambda_{\tilde{P}\left(N_{\uparrow \pi}+N_{\uparrow \bar{\pi}}\right)}\right) \Phi_{\bar{A}}\left(P Q, P^{-1}\right)
\end{aligned}
$$


Now we introduce similarly as in previous case the vector $\hat{\Phi}_{\bar{\pi} \uparrow}(P)$ :

$$
\hat{\Phi}_{\bar{\pi} \uparrow}(P) \equiv \sum_{\bar{A} \in F} \sum_{Q^{\prime} \in \mathfrak{S}_{N_{\bar{\pi} \uparrow}}} \Phi_{\bar{A}}\left(P, Q^{\prime}\right) \prod_{i \in \mathcal{M}_{\bar{\pi} \uparrow}} e_{i}^{\bar{A}_{Q^{\prime}(i)}}
$$

where $F=\operatorname{span}\{(\mathfrak{p}+1) \uparrow, \ldots, \mathfrak{n} \uparrow\}$.

The relations (A.56) and (A.63) can be gathered in $\hat{\Phi}_{\bar{\pi} \uparrow}(P)$ :

$$
\hat{\Phi}\left(\Pi_{a b} P\right)=\alpha_{a b}^{-1} \mathcal{P}_{a b} \hat{\Phi}(P), \quad \text { with } \quad P^{-1}(a)-P^{-1}(b)=-1
$$

where $\mathcal{P}_{a b}$ is the permutation acting on the particles situated on $a$ and $b$ positions in $\hat{\Phi}_{\bar{\pi} \uparrow}(P)$.

Therefore, the periodicity problem can be rewritten as follows:

$$
X_{m} \mathcal{P}_{\tilde{P}\left(N_{\uparrow \pi}+1\right) m} \cdots \mathcal{P}_{\tilde{P}\left(N_{\uparrow \pi}+N_{\uparrow \bar{\pi}}-1\right) m} \hat{\Phi}_{\bar{\pi} \uparrow}(\tilde{P})=\prod_{l=1}^{N} \sigma_{l}\left(\lambda_{m}\right) \hat{\Phi}_{\bar{\pi} \uparrow}(\tilde{P})
$$

with $m=\tilde{P}\left(N_{\uparrow \pi}+N_{\uparrow \bar{\pi}}\right)$ and

$$
X_{m}=\prod_{l \in \mathcal{M}_{\bar{\pi} \uparrow}, l \neq m} \alpha_{m l} \prod_{i \in \mathcal{M}_{\pi \downarrow}} e^{i k_{a_{i}}} \sigma_{a_{i}}\left(\lambda_{m}\right) \prod_{i \in \mathcal{M}_{\bar{\pi} \downarrow}} b_{i}^{-1} \prod_{i \in \mathcal{M}_{\pi \uparrow}} e^{-i k_{a_{i}}} \sigma_{a_{i}}\left(\lambda_{m}\right)
$$

Choosing $\tilde{P}=C_{\bar{\pi} \uparrow}^{-m}$ with $C_{\bar{\pi} \uparrow}=\Pi_{N_{\uparrow \pi}+N_{\uparrow \bar{\pi}}}^{N_{\uparrow \pi}+1} \ldots \Pi_{N_{\uparrow \pi}+N_{\uparrow \bar{\pi}}}^{N_{\uparrow \pi}+N_{\uparrow \bar{\pi}}-1}$, we find the Bethe equations:

$\prod_{l \in \mathcal{M}_{\bar{\pi} \uparrow}, l \neq m} \alpha_{m l} \prod_{i \in \mathcal{M}_{\pi \downarrow}} e^{i k_{a_{i}}} \sigma_{a_{i}}\left(\lambda_{m}\right) \prod_{i \in \mathcal{M}_{\bar{\pi} \downarrow}} b_{i}^{-1} \prod_{i \in \mathcal{M}_{\pi \uparrow}} e^{-i k_{a_{i}}} \sigma_{a_{i}}\left(\lambda_{m}\right) e^{\frac{2 \pi i}{N_{\uparrow} \sum_{i=1}^{N_{\uparrow \bar{\pi}}-N_{\uparrow \mathfrak{n}}} \bar{n}_{i}}}=\prod_{l=1}^{N} \sigma_{l}\left(\lambda_{m}\right)$

with $m \in \mathcal{M}_{\uparrow \bar{\pi}}$ and $1 \leq \bar{n}_{1}<\ldots<\bar{n}_{N_{\uparrow \bar{\pi}}-N_{\uparrow n}} \leq N_{\uparrow \bar{\pi}}$.

The problem of the complete characterization of the eigenfunction and of state counting arises in a similar way as in case III-A. We get additional sets of integers:

$$
1 \leq n_{1}^{(k)}<n_{2}^{(k)}<\ldots<n_{N_{\uparrow(\mathfrak{p}+1)}+\ldots+N_{\uparrow(k-1)}}^{(k)} \leq N_{\uparrow(\mathfrak{p}+1)}+\ldots+N_{\uparrow k}, \quad k=\mathfrak{p}+2, \ldots, \mathfrak{n}-1 .
$$

Again, we recover relation (A.84) if we take $k=\mathfrak{n}$ in the above condition.

Open Access. This article is distributed under the terms of the Creative Commons Attribution Noncommercial License which permits any noncommercial use, distribution, and reproduction in any medium, provided the original author(s) and source are credited.

\section{References}

[1] J. Hubbard, Electron Correlations in Narrow Energy Bands, Proc. Roy. Soc. London A 276 (1963) 238.

[2] J. Hubbard, Electron Correlations in Narrow Energy Bands II. The Degenerate Band Case, Proc. Roy. Soc. London A 277 (1964) 237. 
[3] M.C. Gutzwiller, Effect of Correlation on the Ferromagnetism of Transition Metals, Phys. Rev. Lett. 10 (1963) 15.

[4] A. Montorsi, The Hubbard Model, World Scientific, Singapore (1992).

[5] F. Eßler, H. Frahm, F. Goehmann, A. Klumper and V. Korepin, The One-Dimensional Hubbard Model, Cambridge University Press, Cambridge U.K. (2005).

[6] E.H. Lieb and F.Y. Wu, Absence of Mott transition in an exact solution of the short-range, one-band model in one dimension, Phys. Rev. Lett. 20 (1968) 1445 [SPIRES].

[7] E.H. Lieb and F.Y. Wu, The one-dimensional Hubbard model: a reminiscence, Physica A 321 (2003) 1 [cond-mat/0207529].

[8] B.S. Shastry, Infinite conservation laws in the one-dimensional Hubbard model, Phys Rev Lett 56 (1986) 1529.

[9] B.S. Shastry, Exact integrability of the one-dimensional Hubbard model, Phys Rev Lett 56 (1986) 2453.

[10] B.S. Shastry Decorated star triangle relations and exact integrability of the one-dimensional Hubbard model, J. Stat. Phys. 50 (1988) 57.

[11] E. Olmedilla, M. Wadati and Y. Akutsu, Yang-Baxter Relations for Spin Models and Fermion Models, J. Phys. Soc. Japan 56 (1987) 2298.

[12] M. Shiroishi and M. Wadati, Yang-Baxter equation for the R-matrix of the one-dimensional Hubbard model, J. Phys. Soc. Japan 64 (1995) 57.

[13] Z. Maassarani, The SU(N) Hubbard model, Phys. Lett. A 239 (1998) 187 [cond-mat/9709252].

[14] Z. Maassarani, Exact integrability of the $\mathrm{SU}(N)$ Hubbard model, Mod. Phys. Lett. B12 (1998) 51 [cond-mat/9710083].

[15] A. Rej, D. Serban and M. Staudacher, Planar $N=4$ gauge theory and the Hubbard model, JHEP 03 (2006) 018 [hep-th/0512077] [SPIRES].

[16] J.M. Drummond, G. Feverati, L. Frappat and É. Ragoucy, Super-Hubbard models and applications, JHEP 05 (2007) 008 [hep-th/0703078] [SPIRES].

[17] G. Feverati, L. Frappat and É. Ragoucy, Universal Hubbard models with arbitrary symmetry, J. Stat. Mech. (2009) P04014 [arXiv: 0903.0190] [SPIRES].

[18] V. Fomin, L. Frappat and É. Ragoucy, Bethe equations for generalized Hubbard models, JHEP 09 (2009) 055 [arXiv:0906.4512] [SPIRES].

[19] G. Arutyunov, S. Frolov and M. Staudacher, Bethe ansatz for quantum strings, JHEP 10 (2004) 016 [hep-th/0406256] [SPIRES].

[20] T. Bargheer, N. Beisert and F. Loebbert, Long-Range Deformations for Integrable Spin Chains, J. Phys. A 42 (2009) 285205 [arXiv:0902.0956] [SPIRES].

[21] M. Takahashi, One-dimensional Hubbard model at finite temperature, Prog. Theor. Phys. 47 (1972) 69.

[22] T. Deguchi, F.H.L. Essler, F. Gohmann, A. Klumper, V.E. Korepin, and K. Kusakabe. Thermodynamics and excitations of the one-dimensional Hubbard model, Phys. Rep. 331 (2000) 197 [cond-mat/9904398].

[23] F. Dolcini and A. Montorsi, Results on the symmetries of integrable fermionic models on chains, Nucl. Phys. B 592 (2001) 563 [cond-mat/0110246].

[24] B. Sutherland, An introduction to the Bethe ansatz, Lect. Note Phys. 242 1985 1, in Exactly Solvable Problems in Condensed Matter and Relativistic Field Theory, B. Shastry, S. Jha and V. Singh eds., Springer, Berlin Germany (1985). 